\title{
Association of Adipose Tissue Inflammation With Histologic Severity of Nonalcoholic Fatty Liver Disease
}

\author{
Johannie du Plessis, ${ }^{1,2, \star}$ Jos van Pelt, ${ }^{1, *}$ Hannelie Korf, ${ }^{3, *}$ Chantal Mathieu, ${ }^{3}$ \\ Bart van der Schueren, ${ }^{3}$ Matthias Lannoo, ${ }^{4}$ Tom Oyen, ${ }^{4}$ Baki Topal, ${ }^{4}$ Gary Fetter, ${ }^{5}$ \\ Simon Nayler, ${ }^{6}$ Tessa van der Merwe, ${ }^{5,7}$ Petra Windmolders, ${ }^{1}$ Luc Van Gaal, ${ }^{8}$ An Verrijken, ${ }^{8}$ \\ Guy Hubens, ${ }^{9}$ Martin Gericke, ${ }^{10}$ David Cassiman, ${ }^{1,11}$ Sven Francque, ${ }^{12}$ Frederik Nevens, ${ }^{1,11}$ \\ and Schalk van der Merwe ${ }^{1,11}$
}

\begin{abstract}
${ }^{1}$ Laboratory of Hepatology, Faculty of Medicine, ${ }^{3}$ Laboratory of Clinical and Experimental Endocrinology, ${ }^{4}$ Department of Abdominal Surgery, University of Leuven, Leuven, Belgium; ${ }^{2}$ Department of Immunology, Hepatology and GI Research Laboratory, ${ }^{7}$ Department of Endocrinology, University of Pretoria, Pretoria, South Africa; ${ }^{5}$ Waterfall City Centre of Excellence, Waterfall City Hospital, Johannesburg, South Africa; ${ }^{6}$ Histopathology, The Wits University Donald Gordon Medical Centre, Johannesburg, South Africa; ${ }^{8}$ Department of Endocrinology, Diabetology and Metabolism, ${ }^{9}$ Department of Abdominal Surgery, ${ }^{10}$ Institute of Anatomy, Leipzig University, Germany; ${ }^{11}$ Department of Internal Medicine, Division of Liver, Gallbladder and Pancreaticobiliary Disorders, University Hospital Gasthuisberg, Leuven; and ${ }^{12}$ Department of Gastroenterology and Hepatology, Antwerp University Hospital, University of Antwerp, Edegem, Belgium
\end{abstract}

BACKGROUND \& AIMS: The prevalence of nonalcoholic fatty liver disease (NAFLD) has increased with the obesity pandemic. We analyzed the transcriptional profiles of subcutaneous adipose tissue (SAT) and visceral adipose tissue (VAT), and phenotypes and functional characteristics of adipocyte tissue macrophages (ATMs), in obese patients undergoing bariatric surgery. METHODS: We collected anthropometric data; plasma samples; and SAT, VAT, and liver tissues from 113 obese patients undergoing bariatric surgery at academic hospitals in Europe (Antwerp and Leuven) and South Africa. Based on clinical and histologic features, patients were assigned to the following groups: obese, NAFLD, nonalcoholic steatohepatitis (NASH), or NASH with fibrosis. Microarray analyses were performed to identify genes expressed differentially among groups. We measured levels of cytokines and chemokines in plasma samples and levels of RNAs in adipose tissues by quantitative reverse-transcription polymerase chain reaction. ATMs were isolated from patients and 13 lean individuals undergoing cholecystectomy (controls), analyzed by flow cytometry, and cultured; immunophenotypes and levels of cytokines and chemokines in supernatants were determined. RESULTS: We observed increased expression of genes that regulate inflammation in adipose tissues from patients with NAFLD and NASH; expression of these genes increased as disease progressed from NAFLD to NASH. We found 111 genes associated with inflammation that were expressed differentially between VAT and SAT. Serum levels of interleukin 8, chemokine (C-C motif) ligand 3 , and tumor necrosis factor- $\alpha$ correlated with liver inflammation and NAFLD activity score. We developed 2 models that could be used to determine patients' liver histology based on gene expression in VAT and SAT. Flow cytometry showed increased proportions of CD11c+CD206+ and CCR2+ macrophages in VAT from patients with NASH, and supernatants of cultured macrophages had increased levels of cytokines and chemokines compared with controls. CONCLUSIONS: VAT and SAT from patients with NAFLD and NASH have an increased expression of genes that regulate inflammation, and ATM produce increased levels of inflammatory cytokines, compared with adipose tissues from controls. We identified an expression profile of 5 genes in SAT that accurately predict liver histology in these patients. Transcript profiling: accession numbers: GSE58979 and GSE59045.

Keywords: Gene Expression; IL8; Immune Regulation; Inflammatory Response.

The incidence of fatty liver disease has lincreased inparallel to the global obesity pandemic and is predicted to become the most important indication for liver transplantation during the next decade. Adipose tissue is a mediator of metabolic and cardiovascular disorders in the general population, and has been implicated in the development of nonalcoholic fatty liver disease (NAFLD). ${ }^{2}$ Abdominal adiposity, quantified by magnetic resonance imaging, correlates with steatosis in healthy individuals and with severity of inflammation and fibrosis in subjects with nonalcoholic steatohepatitis (NASH).

In obesity, adipocytes develop hypertrophy and undergo hyperplasia, enabling these cells to compartmentalize triglycerides. ${ }^{3}$ This containment mechanism ultimately may fail, resulting in hypoxia, adipocyte cell

*Authors share co-first authorship.

Abbreviations used in this paper: ALT, alanine aminotransferase; AST, aspartate aminotransferase; AT, adipose tissue; ATM, adipose tissue macrophage; AUROC, area under receiver operating characteristic; BMI, body mass index; CCL, chemokine (C-C motif) ligand; CDNA, complementary DNA; CCR, C-C chemokine receptor; IL, interleukin; NAFLD, nonalcoholic fatty liver disease; NAS, nonalcoholic fatty liver disease activity score; NASH, nonalcoholic steatohepatitis; PNPLA3, patatin-like phospholipase domain-containing protein 3; qRT-PCR, quantitative reverse transcription polymerase chain reaction; SAT, subcutaneous adipose tissue; TNF, tumor necrosis factor; VAT, visceral adipose tissue. 
death, and enhanced chemokine secretion, which attracts activated macrophages to this site. ${ }^{3}$ In addition, insulinresistant adipocytes release excessive free fatty acids that may induce inflammation in organs such as the liver. ${ }^{4}$ What remains difficult to explain, however, is why only a minority of obese individuals develop NASH or progress to fibrosis and cirrhosis.

Most of the current understanding about the role of macrophages in obesity stems from animal experiments. In mice, obesity is associated with a switch of macrophages to an inflammatory phenotype characterized by the accumulation of CCR2-positive cells in adipose tissue., ${ }^{5,6}$ Ablation of adipose tissue macrophages (ATMs) normalized wholebody insulin sensitivity, confirming the importance of this cell type in the development of insulin resistance. ${ }^{7}$ Chemokine (C-C motif) ligand (CCL)2 knock-out mice, which have a reduced capacity to attract macrophages to adipose tissue, developed less hepatic steatosis and insulin resistance. ${ }^{8}$ Leptin-deficient ob/ob mice that were transfected to overexpress adiponectin remained insulinsensitive despite accumulating more adipose tissue compared with ob/ob littermates. In this model, the improved metabolic profile correlated with a reduced influx in macrophage numbers. ${ }^{9}$ In mice fed a high-fat diet, macrophage activation and proinflammatory gene expression preceded the development of inflammation in the liver. ${ }^{10}$ In this study messenger RNA levels of macrophage and inflammation-associated genes were up-regulated strongly in adipose tissue by week 6 but only developed in liver tissue after 16 weeks. ${ }^{10}$ Collectively, these studies indicated that adipose tissue inflammation, induced by activated ATMs, is a prerequisite for the development of steatohepatitis in mice.

Although the importance of visceral adipose tissue (VAT) inflammation in the development of insulin resistance and steatohepatitis has been established, the role of subcutaneous adipose tissue (SAT) remains less clear. Recently, Tordjman et $\mathrm{al}^{11}$ confirmed the expression of proinflammatory genes using microarray, and the accumulation of macrophages in deep SAT in patients with NASH. In another study, microarray gene expression analysis of SAT showed up-regulation of genes related to innate and adaptive immunity, and macrophage infiltration correlated with hepatic fat content. ${ }^{12}$ Taken together these studies suggest that peripheral adipose tissue also may be important in the pathogenesis of NASH.

We sought to determine the role of adipose tissue in the development of NASH in a large, well-characterized bariatric population, using microarray and quantitative polymerase chain reaction (PCR) analysis to characterize gene expression profiles in subcutaneous and visceral adipose, and liver tissue. This unbiased approach allowed us to determine molecular events in obese individuals stratified, based on liver histology, into distinct well-defined histologic subgroups. Exploiting the fact that variables such as age and body mass index (BMI) were comparable between the groups allowed the comparison of gene expression profiles in individuals with limited liver disease with patients who developed NASH and fibrosis. Our study confirms the importance of adipose tissue inflammation, and has highlighted the role of CD11c+CD206+ macrophages and the secretion of proinflammatory cytokines by ATMs in the pathogenesis of NAFLD. Our study also has identified a gene signature capable of differentiating between the histologic subgroups with a high degree of accuracy.

\section{Materials and Methods}

\section{Study Population}

A cohort study was performed that included severely obese patients undergoing bariatric surgery in 3 academic hospitals from Europe (Antwerp and Leuven) and South Africa. Before surgery all patients were assessed anthropometrically and serum was collected for later analysis. At surgery, VAT and SAT samples were obtained and snap frozen for subsequent RNA extraction. A liver biopsy was performed using a 16G Tru-Cut biopsy needle (BARD, Murray Hill, NJ) for histopathologic assessment and from which RNA was extracted. All patients provided written informed consent and the study protocols were approved by all 3 research institutions.

\section{Liver Histology and Patient Classification}

Liver histology was assessed by expert liver pathologists blinded to all clinical information according to the NASHClinical Research Network Scoring System criteria $^{13}$ (Supplementary Materials and Methods section). Patients with inadequate liver biopsy specimens $(<2 \mathrm{~cm}$ and/or $<5$ portal tracts), with underlying cirrhosis, or borderline features of NASH (NAFLD activity score [NAS], 3-4) were excluded from the analysis.

We subsequently included 113 patients in 4 distinct groups, as follows: obese: less than $5 \%$ steatosis, NAS of 0 ; NAFL: NAFLD without inflammation or NASH, NAS less than 4; NASH: NASH without significant fibrosis, NAS of 5 or greater and fibrosis score of $0-1$; NASH with fibrosis: NASH with a significant fibrosis (score, 2-3), but no cirrhosis.

\section{Study Design}

A total of 113 patients were divided randomly into 2 representative groups: in 35 patients (training cohort) the full spectrum of gene expression in SAT and VAT was investigated using microarray for the identification of marker genes and the assessment of molecular pathways (Figure 1). By using the microarray data a gene set was selected, the expression of these genes was confirmed by quantitative reversetranscription PCR (qRT-PCR) and predictive models for VAT and SAT developed in combination with clinical characteristics of the 35 patients in the training cohort. In the second group of 78 patients (validation cohort) qRT-PCR analysis was performed on the gene set incorporated into the prediction model and the results were used to evaluate the performance of the models for both VAT and SAT. The performance of the models was compared with serum procollagen III and patatin-like phospholipase domain-containing protein 3 (PNPLA3) gene mutation analysis. Microarray analysis of liver tissue also was performed in 15 of the 35 patients with limited liver disease (excluding patients with NASH with fibrosis) to compare gene expression profiles in early NAFLD in liver and adipose tissue. Finally, we included an additional 26 bariatric patients also stratified according to 
Figure 1. The study design. In a training set of 35 patients, visceral and subcutaneous adipose, and liver tissue were profiled by microarray, to study molecular pathways and to select classifier genes. The combination of qRT-PCR data obtained from adipose tissue in the training set and the corresponding biochemical parameters were used to generate prediction models that could classify patients into histologic subgroups accurately. The models generated for visceral adipose and for subcutaneous adipose tissue then were validated using an independent validation set of 78 patients.

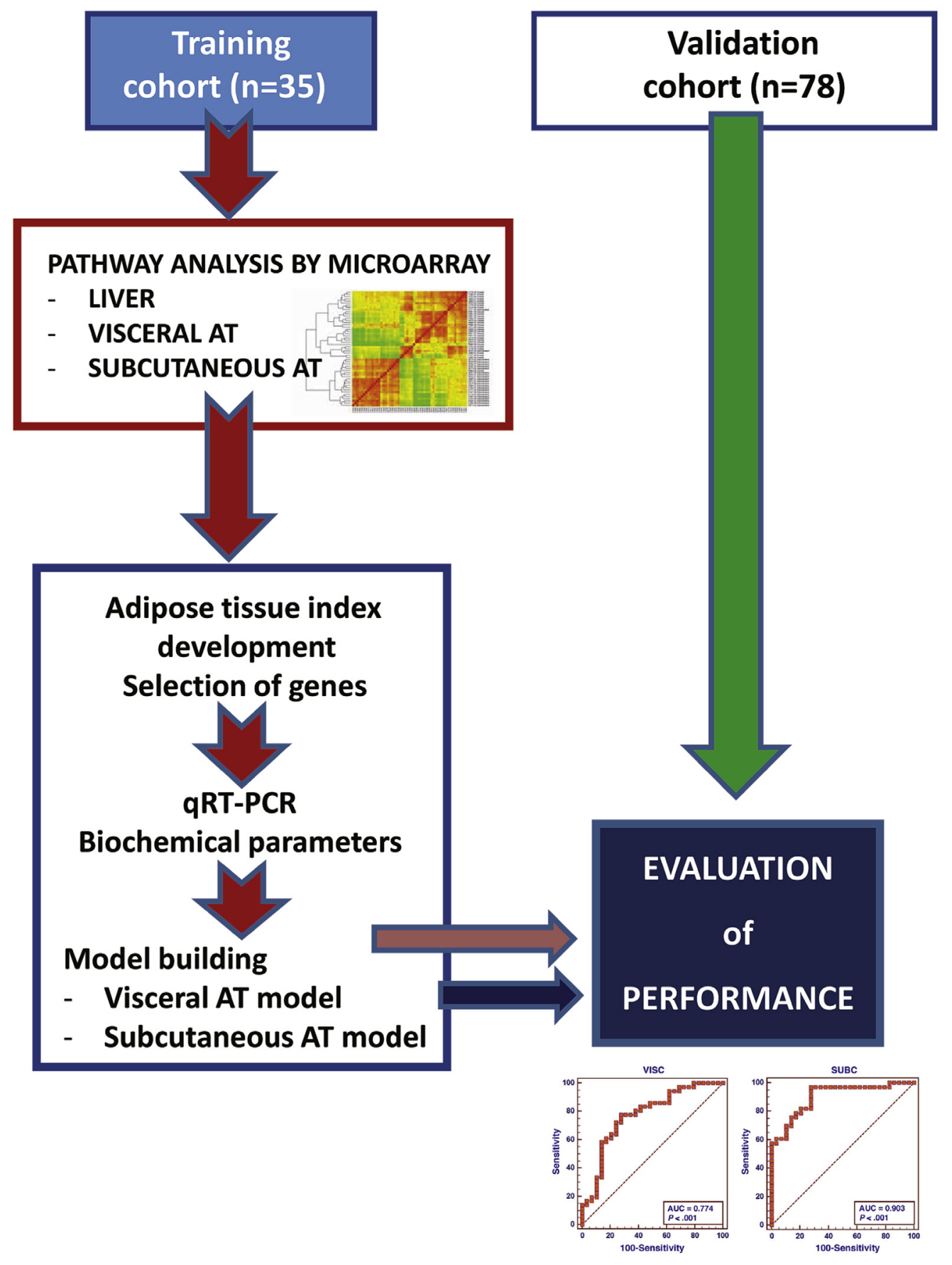

\section{Microarray and Data Processing}

Microarray hybridization was performed on Affymetrix Primeview arrays according to the manufacturer's instructions (Affymetrix, Inc, Santa Clara, CA) (see the Supplementary Materials and Methods section for more detail).

\section{RNA Extraction, Complementary DNA Synthesis,} and Quantitative RT-PCR

Adipose tissue was homogenized, RNA was isolated by means of the RNeasy Kit (Qiagen, Chatsworth, CA), and complementary DNA (cDNA) was synthesized. Quantitative RT-PCR was performed with the A7500 Fast Real-Time PCR System (Applied Biosystems, Foster City, CA) (Supplementary Materials and Methods section).

\section{Pathway Analysis}

Gene enrichment and pathway analysis was performed with the internet-based DAVID Bioinformatics Resource 6.7 program (National Institute of Allergy and Infectious Diseases [NIAID], NIH, Bethesda, MD). ${ }^{14}$ To investigate known and predicted protein-protein interactions for the differentially expressed 
genes we used the String 9.1 program (EMBL, Heidelberg, Germany) $^{15}$ (see the Supplementary Materials and Methods section for more detail).

\section{Biochemical Analysis and Cytokine Measurements}

Blood was collected from a peripheral vein before surgery, centrifuged, and stored at $-80^{\circ} \mathrm{C}$ until further analysis. Standard biochemical analysis was performed in 113 patients. In 78 patients the Custom Meso Scale Discovery V-plex assays (Meso Scale Diagnostics, Rockville, MD) were used to determine plasma cytokine interleukin (IL)1 $\beta$, IL6, IL8, IL10, tumor necrosis factor (TNF) $\alpha$, and chemokines levels (monocyte chemotactic protein 1/CCL2), monocyte chemoattractant protein 4/CCL13, macrophage-derived chemokine, and macrophage inflammatory protein $1 \alpha$. All measurements were performed in duplicate.

\section{Serum Procollagen III N-Terminal Propeptide}

Procollagen III N-terminal Propeptide serum levels were determined in duplicate according the manufacturer's instructions (SEA573Hu; Cloud-Clone Corp, Houston, TX). Lower limits of all assays are shown in see the Supplementary Materials and Methods section.

\section{PNPLA3 Genotyping}

PNPLA3 variant rs738409 was genotyped as previously described. ${ }^{16}$

\section{Model Development and Evaluation}

We identified, from the microarray data set in VAT and SAT, the most differentially expressed genes between the obese and NASH with fibrosis subgroups. By using these data we selected a set of genes for both VAT and SAT that could, by hierarchic clustering, accurately classify the 35 patients in the training cohort to either obese/NAFL or NASH/NASH with fibrosis subgroups. These genes then were selected for model building and subsequently determined by qRT-PCR in all patients. By using the qRT-PCR data from the 35 patients in the training set, as well as 18 clinical and biochemical parameters, a mathematic model was built for both VAT and SAT using MedCalc Statistical Software version 13.1.2 (MedCalc Software bvba, Ostend, Belgium). Models were optimized and an equation was generated that could predict liver histology from a minimal number of parameters. The performance of the models then was investigated in an independent cohort of 78 patients. Blinded for the histologic classification, the regression gene score was calculated, patients were classified into histologic subgroups, and an area under receiver operating characteristic (AUROC) analysis was performed to assess the sensitivity and specificity.

\section{Flow Cytometric Analysis and Culture of VAT Macrophages}

Adipose tissue was processed to obtain a single-cell suspension and preincubated with Fc block to minimize nonspecific binding. Cells $\left(2 \times 10^{5}\right)$ were labeled with the following conjugated antibodies: CD45, CD14, CD11c, HLA-DR, CD33, CD11b (eBioscience, San Diego, CA), CD206 (BD Bioscience Franklin Lakes, NJ), CCR2 (R\&D Systems Minneapolis, MN), or matching isotype controls in $\mathrm{Ca} 2+-$ and $\mathrm{Mg} 2+$-free phosphate- buffered saline containing $2 \mathrm{mmol} / \mathrm{L}$ EDTA and $0.1 \%$ bovine serum albumin. Data acquisition was performed on a Gallios flow cytometer (Beckman Coulter, Analis, Suarlée, Belgium) and analyzed using FlowJo V.10 software (Tree Star, Inc, Ashland, OR). Macrophages were identified based on forward and side scatter as well as double positivity for CD45 and CD14. Additional subsets within this population were evaluated based on CD206 and CD11c co-staining (see the Supplementary Materials and Methods section).

In addition, cells isolated from VAT tissue were resuspended in RPMI medium containing 10\% heat-inactivated fetal calf serum, $100 \mathrm{U} / \mathrm{mL}$ penicillin, $100 \mathrm{mg} / \mathrm{mL}$ streptomycin, and $50 \mathrm{mmol} / \mathrm{L} \beta$-mercaptoethanol. Cells $\left(2 \times 10^{5}\right.$ per well $)$ were seeded in 96 -well tissue culture plates, incubated at $37^{\circ} \mathrm{C}$ for 1 hour before washing away the nonadherent cells, resulting in more than $90 \%$ enrichment for CD14+ within the CD45+ leukocyte gate (data not shown). After an additional 24-hour incubation at $37^{\circ} \mathrm{C}$ the culture media was collected, centrifuged, and stored at $-20^{\circ} \mathrm{C}$. Custom Meso Scale Discovery V-plex assays were used to determine macrophage-derived cytokine and chemokine production.

\section{Immunofluorescence Staining of VAT Sections}

Visceral adipose tissue were fixed immediately in $6 \%$ Paraformaldehyde (PFA) and embedded in paraffin. Adipose tissue (AT) sections (7- $\mu$ m-thick) were deparaffinized and processed for immunofluorescence (see the Supplementary Materials and Methods section).

\section{Statistics}

MedCalc Statistical Software version 13.1.2 (MedCalc Software, Ostend, Belgium; http://www.medcalc.org; 2014) was used for multiple regression analysis, model building, intergroup comparison, and correlation assessment (Bland-Altman and Pearson correlations). Statistical differences between groups were assessed with the Student $t$ test or the Mann-Whitney rank-sum test where appropriate. A $P$ value less than .05 was considered statistically significant.

\section{Results}

\section{Patient Characteristics}

This study was performed in 113 severely obese patients classified according to strict and clinically relevant histologic criteria. The main clinical and biochemical characteristics of the study population are shown in Table 1. There were no differences in age or BMI between the groups. Groups were significantly different in waist circumference, transaminase levels, and high-density lipoprotein cholesterol level in relation to NASH severity. No significant differences were found between the training and the validation cohorts (Supplementary Results section) or between the different institutions (data not shown).

\section{Gene Expression}

Microarray analysis of liver tissue showed that in early NAFLD, comparing obese-to-NAFL with NAFL-to-NASH, pathways associated with steroid, terpenoid backbone, unsaturated fatty acids biosynthesis, and peroxisome 
Table 1.Patient Characteristics: Clinical and Biochemical Characteristics and Plasma Cytokine and Chemokine Levels Across the Histologic Subgroups

\begin{tabular}{|c|c|c|c|c|c|}
\hline Characteristic & $\begin{array}{l}\text { Group 1: obese } \\
\quad(n=28)\end{array}$ & $\begin{array}{l}\text { Group 2: NAFL } \\
\quad(n=25)\end{array}$ & $\begin{array}{l}\text { Group 3: NASH } \\
\quad(n=41)\end{array}$ & $\begin{array}{c}\text { Group 4: NASH } \\
\text { with fibrosis } \\
(\mathrm{n}=19)\end{array}$ & $P$ value \\
\hline Age, $y$ & 39 [36-44] & $42[31.5-47.5]$ & 48 [36.5-55] & $44[28-50]$ & NS \\
\hline Sex, \% male & $14.8 \%$ & $8.0 \%$ & $36.6 \%$ & $42.1 \%$ & .013 \\
\hline $\mathrm{BMI}, \mathrm{kg} / \mathrm{cm}^{2}$ & $40[37-44]$ & $42[40-49]$ & $40[40-44]$ & $41[40-44]$ & NS \\
\hline Waist circumference, $\mathrm{cm}$ & $114[107-126]$ & 122 [115-129] & $122[118-131]$ & $128[121-133]$ & .02 \\
\hline Waist-to-hip ratio & $0.90[0.85-0.98]$ & $0.93[0.9-0.97]$ & $1.03[0.95-1.1]$ & $1.03[0.96-1.12]$ & $<.001$ \\
\hline Fat percentage, $\%$ & $54[51-57]$ & $54[50-57]$ & $49[43-54]$ & $50[45-55]$ & .02 \\
\hline \multicolumn{6}{|l|}{ Biochemical parameters } \\
\hline ALT level, $U / L$ & 24 [21-33] & 28 [22-42] & 38 [26-51] & $51[39-88]$ & $<.001$ \\
\hline AST level, U/L & $21[16-25]$ & 24 [20-29] & 27 [20-34] & 39 [27-88] & $<.001$ \\
\hline ALP level, U/L & $87[71-110]$ & 87 [68-103] & 81 [69-101] & 83 [69-113] & NS \\
\hline GGT level, $U / L$ & 27 [22-38] & 28 [23-33] & $40[31-48]$ & 37 [28-55] & .002 \\
\hline Total cholesterol, $\mathrm{mmol} / \mathrm{L}$ & $5.4[4.4-5.9]$ & $5.0[4.7-5.8]$ & $4.8[4.2-5.7]$ & $5.2[4.7-5.7]$ & NS \\
\hline $\mathrm{HDL}, \mathrm{mmol} / \mathrm{L}$ & $1.3[1.0-1.6]$ & $1.2[1.0-1.4]$ & $1.1[0.9-1.3]$ & $1.0[0.9-1.2]$ & .03 \\
\hline $\mathrm{LDL}, \mathrm{mmol} / \mathrm{L}$ & $3.4[2.8-3.8]$ & $3.2[2.7-4.0]$ & $2.8[2.3-3.5]$ & $3.3[2.9-3.7]$ & NS \\
\hline $\mathrm{TG}, \mathrm{mmol} / \mathrm{L}$ & $1.4[1.1-2.2]$ & $1.5[1.3-1.6]$ & $1.8[1.3-2.4]$ & $1.7[1.3-2.8]$ & NS \\
\hline Fasting glucose, $\mathrm{mmol} / \mathrm{L}$ & $4.5[4.2-5.3]$ & $4.6[4.1-5.3]$ & $4.8[4.4-5.7]$ & $4.9[4.4-5.9]$ & NS \\
\hline $\begin{array}{l}\text { Plasma cytokines and } \\
\text { chemokines, } \mathrm{pg} / \mathrm{mL}\end{array}$ & $\begin{array}{l}\text { Group 1: obese } \\
\quad(n=17)\end{array}$ & $\begin{array}{l}\text { Group 2: NAFL } \\
\quad(n=20)\end{array}$ & $\begin{array}{l}\text { Group 3: NASH } \\
\quad(n=24)\end{array}$ & $\begin{array}{c}\text { Group 4: NASH } \\
\text { with fibrosis } \\
(n=16)\end{array}$ & \\
\hline IL1 $\beta$ & $0.008[0-0.28]$ & $0.018[0-0.2]$ & $0.02[0-0.7]$ & $0.01[0-0.05]$ & NS \\
\hline IL6 & $0.5[0.2-7.5]$ & $0.4[0.3-1.1]$ & $0.5[0.07-1.7]$ & $0.53[0.03-1.7]$ & NS \\
\hline IL8 & $1.6[0.8-3.3]$ & $1.4[0.8-2.6]$ & $1.7[1-4.1]$ & $2.9[0.7-5.8]$ & .03 \\
\hline IL10 & $0.06[0.02-0.9]$ & $0.06[0.01-0.3]$ & $0.09[0.01-0.2]$ & $0.1[0.06-0.13]$ & NS \\
\hline $\mathrm{TNF} \alpha$ & $1.1[0.8-1.9]$ & $1.0[0.8-1.4]$ & $1.2[0.9-1.5]$ & $1.4[1-3.2]$ & .001 \\
\hline MCP-1/CCL2 & 163 [104-235] & 182 [93-439] & $191[40-420]$ & $178[28-418]$ & NS \\
\hline MCP-4/CCL13 & $5.7[2.4-24.4]$ & $6.7[3.3-20.5]$ & $8.7[2.8-51.6]$ & $8.6[2.1-29.9]$ & NS \\
\hline MDC & 634 [429-1380] & $715[411-1267]$ & $673[329-1834]$ & 857 [429-1874] & NS \\
\hline MIP- $1 \alpha / \mathrm{CCL} 3$ & $6.5[4.8-10.2]$ & 5.9 [3.1-8.5] & 6.7 [5.2-9.8] & 8.9 [3.8-15.2] & .007 \\
\hline
\end{tabular}

NOTE. Results are expressed as median and interquartile range [25\%-75\%], the Kruskal-Wallis test was used to compare groups and $P<.05$ was considered significant.

ALP, alkaline phosphatase; GGT, $\gamma$-glutamyltransferase; HDL, high-density lipoprotein; LDL, low-density lipoprotein; MCP-1, macrophage chemotactic protein 1; MCP-4, macrophage chemoattractant protein 4; MDC, macrophage-derived chemokine; MIP, macrophage inflammatory protein; TG, triglyceride.

proliferator-activated receptor signaling were up-regulated strongly (see the Supplementary Results section). Although up-regulation of isolated inflammatory genes was noted, no pathways associated with inflammation were expressed differentially. In adipose tissue the number of genes significantly differentially expressed between the histologic stages $(2 \log$ ratio $>1$ or $<-1$ ) increased in VAT with disease progression. In SAT, the number of differentially expressed genes between the groups was less pronounced (Figure $2 A$ ) and increased mainly when comparing NASH with NASH and fibrosis. By using DAVID Bioinformatics we detected no important pathways in VAT when comparing obese-to-NAFL subgroups. In contrast, when comparing NAFL-to-NASH, pathways related to cytokine and chemokine signaling were prominent and increased in complexity with the development of NASH with fibrosis. The complexity of the interactions was visualized using String, which showed the known and predicted protein-protein interactions for the differentially expressed genes. Central molecules that were identified included $I L 8, C C L 2$, jun B proto-oncogene, IL6, and prostaglandin-endoperoxide synthase 2 (Figure 2C).

Prominent pathways identified in SAT related to immune networks, microbiological infections, and cell adhesion. In obese and NAFL the most differentially expressed genes were $I L 6$, prostaglandin-endoperoxide synthase 2, and FBJ murine osteosarcoma viral oncogene homolog, whereas in NASH and NASH with fibrosis up-regulation of CDH1, SERPINE1, apolipoprotein A1, and HLA subtypes were detected (Figure $2 B$ ). Results of the pathway analysis and molecular processes are shown in the Supplementary Results section.

\section{Serum Levels of Proinflammatory Cytokines and Chemokines}

After the observation that the expression of inflammation-related genes was up-regulated prominently in adipose tissue, we subsequently evaluated serum levels of 9 cytokines and chemokines. Serum was available from a 
A

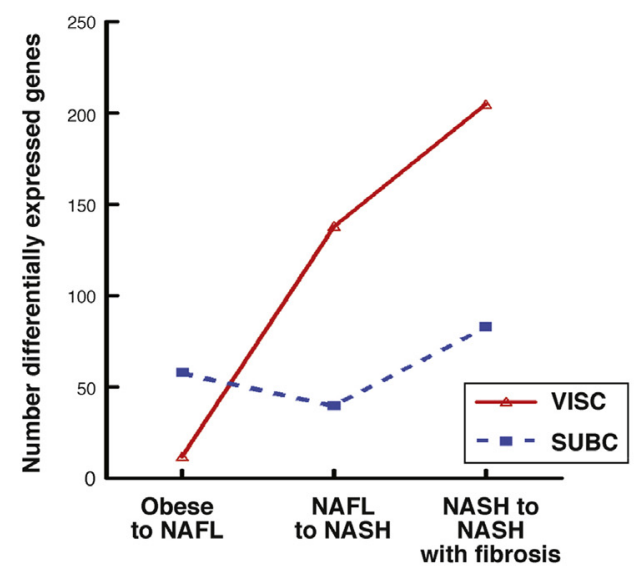

B SUBCUTANEOUS

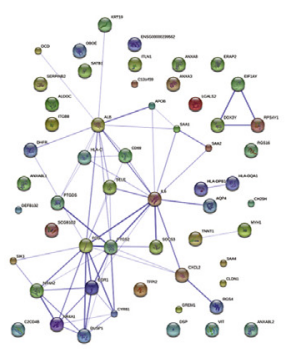

Obese to NAFL

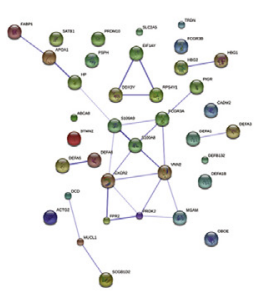

NAFL to NASH NASH to NASH with fibrosis

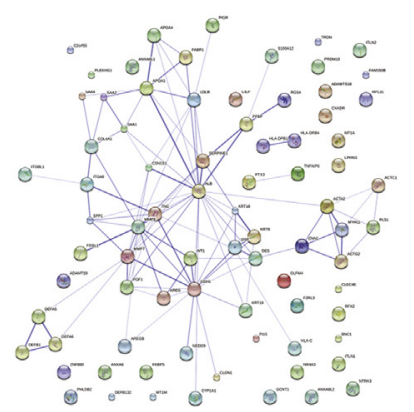

C

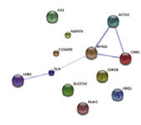

Obese to NAFL
VISCERAL

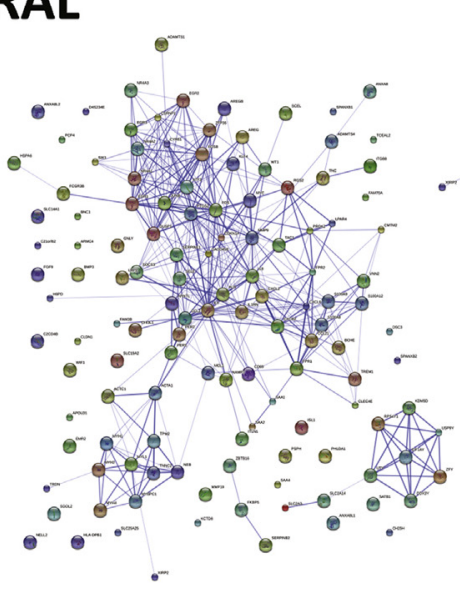

NAFL to NASH

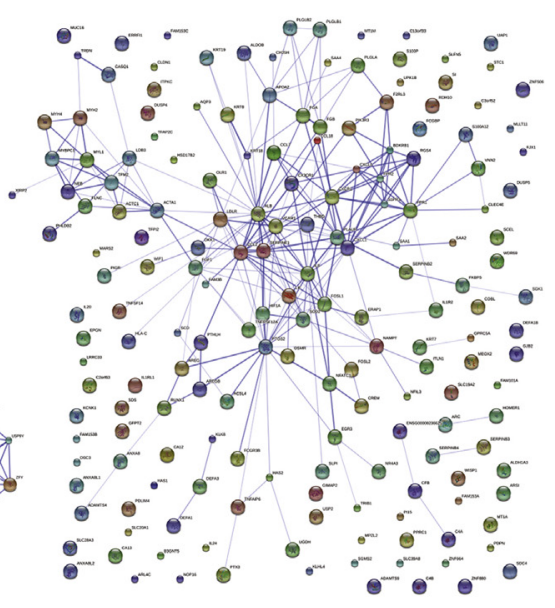

NASH to NASH with fibrosis

Figure 2. Microarray results generated from adipose tissue samples. (A) The number of genes that significantly were expressed differentially (2logratio $>1$ or $<-1$ ) as determined by microarray analysis between the histologic subgroups (solid line, VAT; dashed line, SAT). Graphic representation of the molecular interactions of the differentially expressed genes in $(B)$ SAT and $(C)$ VAT during progression from obese-to-NASH with fibrosis using the String 9.1 computer program.

subset of 76 patients, equally distributed over the 4 classes. Results are listed in Table 1. Significantly increased levels of IL8, TNF $\alpha$, and CCL3 were detected with disease progression that correlated strongly with the NAS score and aspartate aminotransferase (AST) and alanine aminotransferase (ALT) levels (Figure $3 A-D$ ).

\section{Correlation of Inflammatory Gene Expression} Between SAT and VAT

Based on the microarray, hierarchical clustering, and qRT-PCR, there were 9 inflammatory genes (CCL2, CCL3, CCL21, GADD45B, IL1RN, IL8, JUN, RELN, and SERPINE1) selected for analysis in both SAT and VAT. 
A

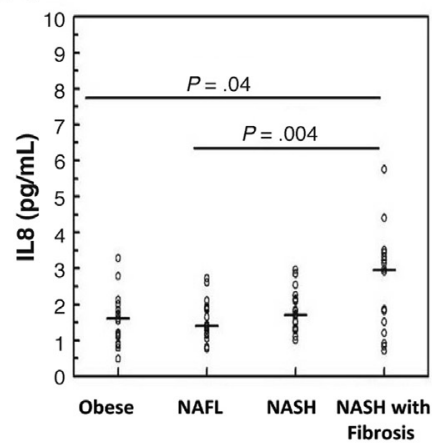

B

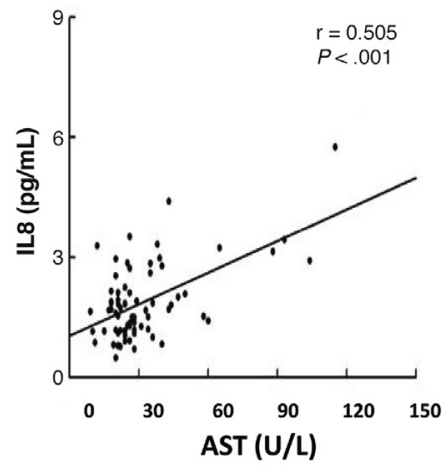

C

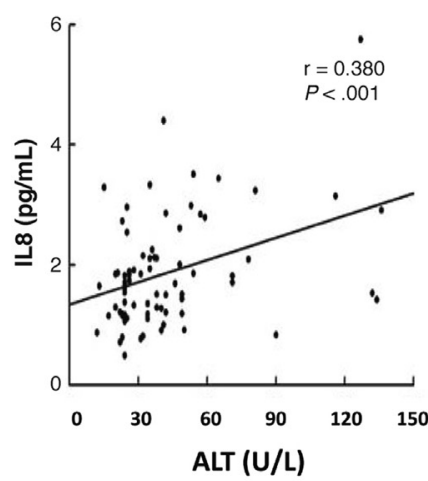

D

Figure 3. $(A-D)$ Correlation between IL8, TNF $\alpha$, and CCL3 plasma levels with liver histology. Significantly increased plasma levels of IL8, TNF $\alpha$ and CCL3 were detected with disease progression (Figure $3 A$ ). Plasma IL8, TNF alpha and CCL3 levels correlated significantly with AST (Figure 3B), ALT (Figure 3C) levels and NAS (Figure $3 D$ ).

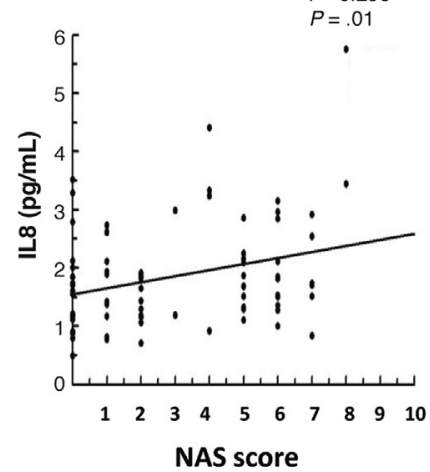

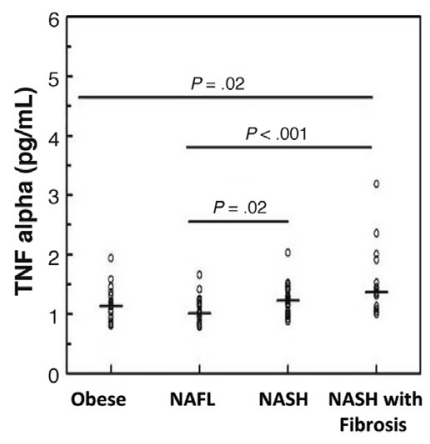
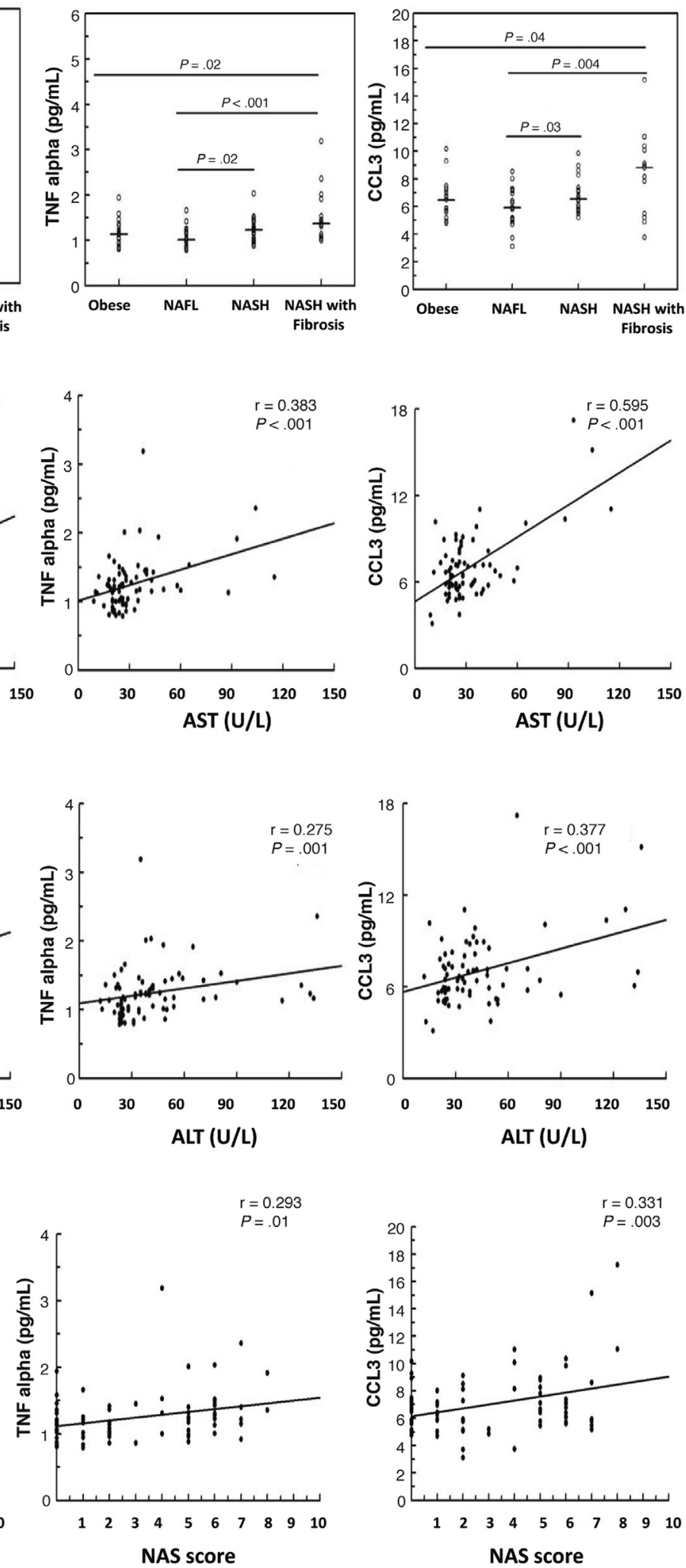

The expression results and fold regulation from qRT-PCR are summarized in Table 2 . We detected a significant positive correlation between all the genes expressed in

VAT and SAT except for CCL21 and CCL3 (see the Supplementary Results section). Bland-Altman analysis showed a higher expression of GADD $45 B$ and CCL21 in SAT 
Table 2. Gene Expression Results of Selected Genes for Both VAT and SAT as Determined by qRT-PCR

\begin{tabular}{|c|c|c|c|c|c|c|c|c|c|c|}
\hline $\begin{array}{l}\text { Gene } \\
\text { symbol }\end{array}$ & $\Delta \mathrm{CT}$ obese & $\Delta \mathrm{CT}$ NAFL & $\Delta \mathrm{CT} \mathrm{NASH}$ & $\Delta \mathrm{CT} N+\mathrm{F}$ & $\begin{array}{c}\text { Fold }^{a} \text { NAFL } \\
\text { vs obese }\end{array}$ & $P$ value & $\begin{array}{l}\text { Fold }^{a} \text { NASH } \\
\text { vs obese }\end{array}$ & $P$ value & $\begin{array}{c}\text { Fold }^{a} \mathrm{~N}+\mathrm{F} \\
\text { vs obese }\end{array}$ & $\begin{array}{c}P \\
\text { value }\end{array}$ \\
\hline \multicolumn{11}{|c|}{ Visceral adipose tissue: $q R T-P C R(n=113)$} \\
\hline CCL2 & $3.3 \pm 2.0$ & $2.9 \pm 1.8$ & $2.1 \pm 1.5$ & $1.9 \pm 1.2$ & 1.3 & NS & 2.3 & .004 & 2.6 & .008 \\
\hline CCL21 & $3.3 \pm 1.5$ & $4.0 \pm 1.5$ & $4.0 \pm 1.3$ & $3.7 \pm 1.1$ & 0.7 & NS & 0.7 & NS & 0.8 & NS \\
\hline CCL3 & $10.2 \pm 1.2$ & $10.0 \pm 0.9$ & $9.4 \pm 1.3$ & $9.8 \pm 1.1$ & 1.1 & NS & 1.8 & .002 & 1.3 & NS \\
\hline GADD45B & $3.3 \pm 2.0$ & $3.2 \pm 2.0$ & $2.2 \pm 1.4$ & $1.5 \pm 1.0$ & 1.1 & NS & 2.3 & .005 & 3.7 & .001 \\
\hline IL1RN & $5.9 \pm 1.4$ & $5.6 \pm 1.7$ & $4.9 \pm 1.4$ & $5.4 \pm 1.2$ & 0.8 & NS & 2.0 & .002 & 0.7 & NS \\
\hline IL8 & $7.8 \pm 2.6$ & $7.6 \pm 3.0$ & $6.3 \pm 2.2$ & $5.3 \pm 2.6$ & 1.1 & NS & 2.8 & .009 & 5.7 & .004 \\
\hline JUN & $6.5 \pm 1.0$ & $6.8 \pm 1.0$ & $6.8 \pm 1.0$ & $7.5 \pm 0.8$ & 0.8 & NS & 0.8 & NS & 0.5 & NS \\
\hline RELN & $6.8 \pm 1.2$ & $7.4 \pm 1.0$ & $7.3 \pm 1.1$ & $7.2 \pm 0.8$ & 0.8 & NS & 0.8 & NS & 0.5 & NS \\
\hline SERPINE & $7.7 \pm 2.0$ & $7.3 \pm 2.1$ & $6.1 \pm 1.6$ & $5.2 \pm 1.4$ & 1.3 & NS & 3.2 & $<.001$ & 6.7 & $<.001$ \\
\hline \multicolumn{11}{|c|}{ Subcutaneous adipose tissue: qRT-PCR $(n=113)$} \\
\hline CCL2 & $2.9 \pm 1.4$ & $2.0 \pm 0.8$ & $2.5 \pm 1.6$ & $1.6 \pm 1.1$ & 1.9 & .02 & 1.2 & NS & 2.5 & .003 \\
\hline CCL21 & $7.4 \pm 1.5$ & $7.0 \pm 1.5$ & $7.2 \pm 1.2$ & $8.2 \pm 1.5$ & 1.2 & NS & 1.1 & NS & 0.5 & NS \\
\hline DMRT2 & $8.8 \pm 0.7$ & $9.0 \pm 0.9$ & $9.5 \pm 1.0$ & $10.1 \pm 0.8$ & 0.9 & NS & 0.6 & .002 & 0.4 & $<.001$ \\
\hline GADD45B & $3.9 \pm 1.5$ & $3.8 \pm 0.9$ & $3.1 \pm 1.0$ & $1.2 \pm 0.7$ & 1.1 & NS & 1.7 & NS & 6.5 & $<.001$ \\
\hline IL1RN & $5.2 \pm 1.2$ & $4.3 \pm 1.0$ & $4.5 \pm 1.3$ & $5.0 \pm 1.1$ & 1.8 & .007 & 1.6 & .04 & 1.1 & NS \\
\hline IL8 & $5.5 \pm 2.7$ & $4.2 \pm 1.7$ & $5.0 \pm 1.8$ & $4.6 \pm 1.9$ & 2.5 & .05 & 1.2 & NS & 1.7 & NS \\
\hline JUN & $5.7 \pm 1.2$ & $5.8 \pm 1.1$ & $6.0 \pm 1.1$ & $6.9 \pm 0.5$ & 0.97 & NS & 0.9 & NS & 0.4 & NS \\
\hline RELN & $8.2 \pm 1.1$ & $8.7 \pm 1.5$ & $7.9 \pm 0.8$ & $8.4 \pm 1.0$ & 1.4 & NS & 0.8 & NS & 1.2 & NS \\
\hline SERPINE & $6.9 \pm 2.1$ & $5.9 \pm 1.7$ & $6.0 \pm 1.4$ & $5.5 \pm 1.2$ & 1.9 & .05 & 1.8 & .06 & 2.5 & .01 \\
\hline ZNF880 & $4.6 \pm 0.7$ & $4.5 \pm 0.9$ & $4.5 \pm 0.9$ & $5.6 \pm 0.5$ & 1.1 & NS & 1.0 & NS & 0.5 & $<.001$ \\
\hline
\end{tabular}

NOTE. Messenger RNA was isolated from SAT. $\triangle$ CT vs B2M. Results are expressed relative to expression levels in obese patients.

$\mathrm{N}+\mathrm{F}$, NASH with fibrosis, (Fold is relative to expression in obese, expressed as \%)

${ }^{a}$ Fold is the difference between obese and the condition: $1=100 \%$ level of expression in obese and $1.2=120 \%=20 \%$ increase vs obese, same $0.7=70 \%=30 \%$ decrease.

compared with VAT, whereas the expression of CCL2, $I L 1 R N, I L 8, J U N$, smf RELN were similar in both adipose tissue compartments (see the Supplementary Results section).

\section{Model Development: Selection of Genes for Classification}

Next we investigated whether gene expression in VAT or SAT, either in isolation or in combination with biochemical and clinical parameters, could be used to classify patients into histologic subgroups. There was a considerable overlap in the differentially expressed genes by microarray between the 2 most extreme subgroups: obese vs NASH with fibrosis for VAT (517 genes) and SAT (140 genes) (Figure 4A). Pathway analysis showed that genes common to both adipose tissue compartments were involved mainly in inflammation (Figure $4 C$ ). We selected 2 sets of genes from the original microarray analysis that were expressed most differentially between obese compared with NASH with fibrosis subgroups for VAT and SAT, and used these to classify 35 patients from the training cohort into clinically important histologic subgroups (obese/NAFL or NASH/NASH with fibrosis) using hierarchic clustering (Figure 4B). This approach also was accurate in assigning patients to intermediate subgroups (NAFL and NASH) and was considered proof of concept for further model development.

\section{Performance of the Predictive Models for VAT and SAT}

Gene expression analysis of the selected genes (see the Supplementary Results section) then was performed by qRT-PCR in all patients in the training and validation cohorts. A logistic regression model that was developed in the training cohort, using the qRT-PCR data from 9 genes and 18 clinical/biochemical parameters, was most predictive in correctly assigning individuals to the clinically relevant subgroups in VAT. This resulted in a model that included AST, BMI, high-density lipoprotein, and the genes CCL2, GADD 45B, and SERPINE1. When this model was validated in a set of 78 patients a good performance was observed (AUROC, 0.774; cut-off value, 0.200; sensitivity, 77.78\%; specificity, $72.40 \%$ ) (Figure 4). In SAT a linear regression model yielded the highest performance predicting liver histology, which was developed incorporating qRT-PCR data from 11 genes and 18 clinical/biochemical parameters. The final model included 5 genes: CCL2, DMRT2, GADD45B, $I L 1 R N$, and IL8. The performance of the model for SAT to assign patients from the validation cohort accurately to the histologic subgroups was excellent (AUROC, 0.903; cut-off value, 2.028; sensitivity, 96.97\%; specificity, $72.41 \%$ ) (Figure $4 D$ ). The exact formula to calculate the regression scores is shown in Figure $4 E$.

Recently, the N-terminal peptide of procollagen III was found to be a sensitive and specific marker to predict liver histology in NAFLD. ${ }^{17}$ The performance of this marker was 
Figure 4. The differential gene expression and receiver operating characteristics (AUROC) curve analysis in visceral and subcutaneous adipose tissue. $(A)$ Microarray analysis of differentially expressed genes between obese and NASH with fibrosis in VAT (517 genes) and SAT (140 genes). There were 111 genes that were expressed differentially in both VAT and SAT that were used for pathways analysis. All differentially expressed genes were used to select classifier genes used in subsequent model building. ( $B)$ Two sets from the most differentially expressed genes in the microarray analysis were identified and hierarchic clustering was performed to classify patients from the training cohort into clinically important histologic subgroups: obese/ $\mathrm{NAFL}$ vs NASH/NASH with fibrosis. The boxes indicate the cluster enriched with patients from the subgroup NASH with fibrosis. This classification was considered proof of concept and the gene expression by qRT-PCR of these selected genes was used for model development. (C) Pathway analysis was performed on 111 genes using the DAVID Bioinformatics Resource 6.7 program to identify molecular mechanisms common to both adipose tissue compartments linking the 111 genes to 6 pathways. (D) Receiver operating characteristics (AUROC) curve analysis performed on 78 VAT and SAT samples in the validation cohort resulting in high statistical significance for VAT (sensitivity, $77.78 \%$; specificity, $72.40 \%$ ) and SAT (sensitivity, 96.97\%; specificity, $72.41 \%$ ), respectively. (E) The formulas to calculate the most representative model for VAT and SAT are shown. Gene expression is used as the $\Delta \Delta \mathrm{Ct}$ value relative to $\beta 2$-microglobulin.
A

B

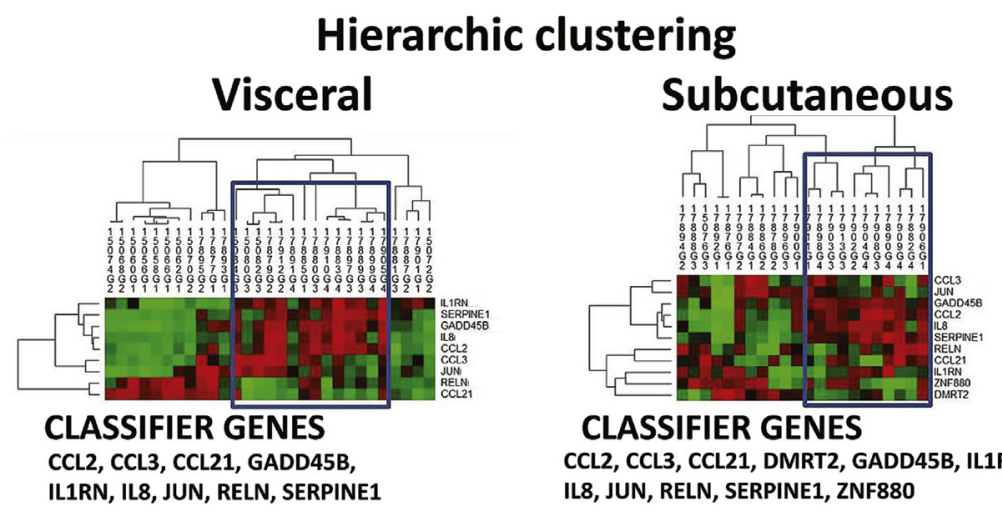

Hierarchic clustering

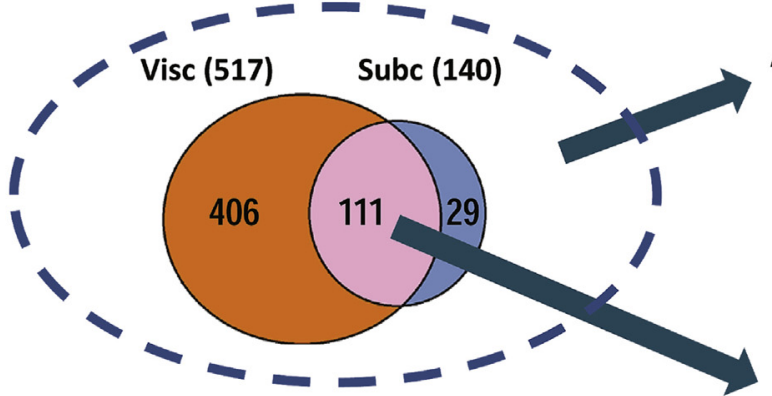

All genes: selection of classifier genes and model building

111 genes overlaping: Identification of joint molecular pathways

CLASSIFIER GENES

CCL2, CCL3, CCL21, DMRT2, GADD45B, IL1RN, IL8, JUN, RELN, SERPINE1, ZNF880

$P$ value

Pathways for 111 genes differentially expressed in both visceral and subcutaneous adipose tissue

\begin{tabular}{|c|c|c|c|}
\hline KEGG PATHWAY & Count & $P$ value & Gene list \\
\hline NOD-like receptor signaling pathway & 7 & 1,10E-05 & $\begin{array}{l}\text { CXCL1, IL6, CCL2, IL8, CXCL2, NFKBIA, } \\
\text { TNFAIP3 }\end{array}$ \\
\hline Cytokine-cytokine receptor interaction & 8 & 0058 & $\begin{array}{l}\text { CXCL1, IL1R2, IL6, CCL2, IL8, TNFRSF12A, } \\
\text { CXCL3, CXCL2 }\end{array}$ \\
\hline $\begin{array}{l}\text { Epithelial cell signaling in } \\
\text { Helicobacter pylori infection }\end{array}$ & 4 & .0193 & CXCL1, IL8, NFKBIA, HBEGF \\
\hline Chemokine signaling pathway & 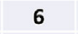 & .0198 & CXCL1, CCL2, IL8, CXCL3, CXCL2, NFKBIA \\
\hline MAPK signaling pathway & 7 & .0235 & $\begin{array}{l}\text { DUSP5, FOS, IL1R2, DUSP1, NR4A1, } \\
\text { GADD45B, MYC }\end{array}$ \\
\hline ErbB signaling pathway & 4 & 0365 & CDKN1A, HBEGF, AREGB, AREG, MYC \\
\hline
\end{tabular}
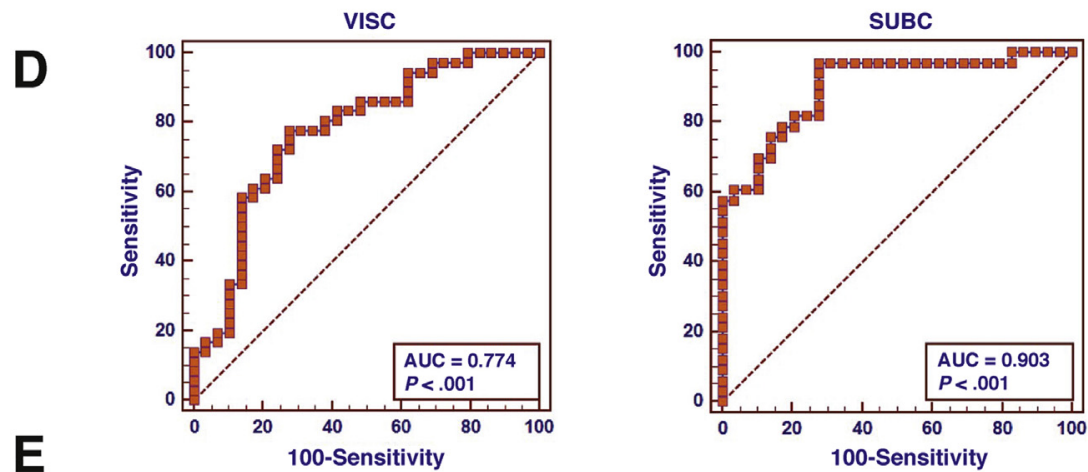

VISC $=6.745+0.042 *$ AST $-0.0018 *$ BMI $-1.471 *$ HDL-0.270*CCL2+0.228*GADD45B-0.906*SERPINE1 SUBC $=1.171+0.253 *$ CCL2+0.218*DMRT2-0.641*GADD45B-0.129*IL1RN $+0.278 *$ IL8

$\operatorname{AST}(\mathrm{U} / \mathrm{L}), \mathrm{BMI}\left(\mathrm{kg} / \mathrm{cm}^{2}\right), \mathrm{HDL}$ (mmol/L), gene expression: ( $\Delta \mathrm{Ct}=\mathrm{nr}$ cycle gene of interest $\mathrm{vs} \mathrm{nr}$ of cycle $\beta$-2-microglobulin) 
evaluated in the subset of 76 patients from whom serum samples were available. AUROC analysis showed an AUROC of 0.692 , sensitivity of $69.23 \%$, and specificity of $67.57 \%$ using a cut-off value of $85 \mathrm{pg} / \mathrm{mL}$ (see the Supplementary Results section). In addition, mutation analysis of the genetic marker PNPLA3_rs738409 was performed to investigate whether it could discriminate between obese/NAFL and NASH/NASH and fibrosis subgroups. A grouping between wild-type vs heterozygote/homozygote for this mutation resulted in an AUROC of 0.691 , a sensitivity of $56.76 \%$, and a specificity of $77.78 \%$ (see the Supplementary Results section).

\section{Adipose Tissue Macrophages From VAT in NASH Secrete Increased Levels of Proinflammatory Cytokines}

We showed that the number of ionized calcium binding adaptor molecule 1 positive (IBA+) ATMs was increased significantly in VAT in all histologic subgroups compared with lean cholecystectomy controls. Neither the number of IBA + ATMs nor the number of crown-like structures, however, differed between the groups (Figure $5 A$ and $B$ ). Fluorescence-activated cell sorter analysis was performed to characterize the immunophenotype of ATMs further. ATMs were identified within the CD45+ leukocyte gate as CD14+ cells and were characterized further with antibodies to CD11c, CD206, and CCR2 (Figure 5C). We detected an increase in the frequency of CD11c+CD206+ as well as CD11c+CCR2 + cells in NASH compared with lean cholecystectomy controls and obese/NAFL subgroups (Figure $5 C$ ). Furthermore, analysis of the supernatant of macrophage cultures showed that ATMs in patients with NASH produced significantly more proinflammatory cytokines/chemokines compared with cholecystectomy controls or obese/NAFL subgroups (Figure $5 D$ ). Collectively, our data suggest that an expansion of CD11c+CD206+ and CCR2+ macrophage populations and the production of proinflammatory cytokines are important in NAFL-to-NASH progression.

\section{Discussion}

Animal studies have shown that adipose tissue inflammation, driven by activated macrophages, is of cardinal importance in the development of NASH. ${ }^{18}$ Genetically manipulating the macrophage phenotype or reducing the influx of activated macrophages into adipose tissue resulted in the preservation of insulin sensitivity and reduced triglyceride accumulation in hepatocytes in these models. ${ }^{7,8,19}$ We hypothesized that adipose tissue inflammation mediated by ATMs also may be a prerequisite in the development of NASH in human beings.

In this study we investigated the role of adipose tissue inflammation in NASH progression by analyzing the full transcriptional profile of VAT and SAT in well-characterized severely obese individuals undergoing bariatric surgery using a microarray approach. Implementing such an unbiased approach and in-depth analysis of differentially expressed genes allowed the identification of unique molecular interactions in VAT and SAT. First, we showed that the number of differentially expressed genes in adipose tissue increased progressively across the histologic subgroups that paralleled disease progression. Second, we showed that pathways associated with cytokine and chemokine signaling were not expressed in VAT in obese patients who had normal liver histology but became apparent in patients with NAFL and more pronounced and complex in NASH and NASH with fibrosis. The central molecules identified in these pathways were $I L 8, C C L 2$, jun B protooncogene, and IL6, all of which are involved in inflammation. The number of differentially expressed genes and pathways in SAT were less pronounced and increased mainly with the development of NASH and fibrosis. When collectively considered, 111 genes were expressed differentially in both VAT and SAT. Pathway analysis of these 111 genes confirmed that they were involved in inflammation, chemokine signaling, and cytokine-chemokine-receptor interaction. Pearson and Bland-Altman correlations showed that gene expression in both of these compartments changed by the same order of magnitude, suggesting that both VAT and SAT are involved, through similar mechanisms, in the pathogenesis of NAFLD-to-NASH progression.

By using an unbiased microarray approach and confirming the results using qRT-PCR our analysis showed the importance of inflammatory cytokines originating from VAT and SAT in the pathogenesis of NASH. Of note, in the absence of inflammatory gene expression in adipose tissue, no histologic abnormalities were observed in the liver, and inflammatory pathways were not expressed at the transcriptional level in liver tissue. Conversely, increased expression of inflammatory genes in adipose tissue correlated with progression from NAFL-to-NASH and fibrosis. These findings strongly support the role of adipose tissue inflammation in the pathogenesis of NASH in human beings. Our study further confirmed the importance of certain cytokines such as CCL2, TNF $\alpha$, and IL1 $\beta$ observed in previous animal and human studies, but also identified novel pathways associated with IL8 and CCL3.

To further extend the results beyond gene expression analysis we assessed the serum levels of inflammatory cytokines and chemokines in a subgroup of 76 individuals. We found that TNF $\alpha$, IL8, and CCL3 plasma levels differed significantly between the groups in keeping with a low-grade inflammatory state. Specifically, increased levels of $\mathrm{TNF} \alpha$, IL8, and CCL3 correlated with ALT and AST levels, and the degree of steatohepatitis as expressed by NAS, strongly suggesting that these cytokines/chemokines are contributing to the pathogenesis of NASH. Levels in serum are a composite of the release of these cytokines/chemokines at various sites including liver and adipose tissue. Microarray analysis of liver tissue in our, as well as other, studies ${ }^{20}$ confirmed that inflammatory pathways are not up-regulated in early NAFL/ NASH, suggesting that cytokines/chemokines in early NAFL/ NASH originate from mainly nonhepatic sources. However, with disease progression from NASH to NASH with fibrosis inflammation in the liver increases as a result of activation of Kupffer and hepatic stellate cells, as well as the infiltration of neutrophils from the circulation. ${ }^{21}$ The resultant secretion of 


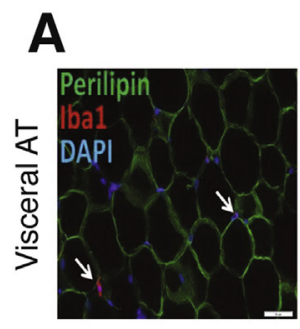

Lean

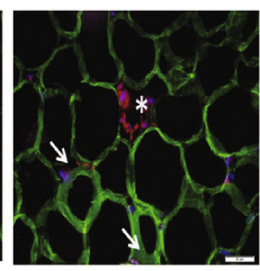

Obese

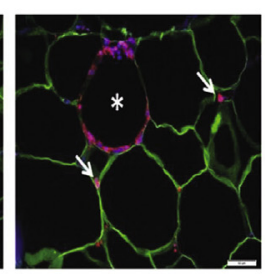

NAFL

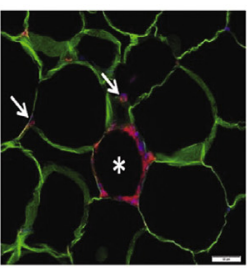

$\mathrm{NASH}$

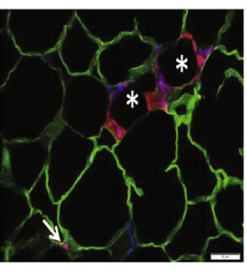

$\mathrm{NASH}+$ fibrosis
Figure 5. Macrophage phenotype and functional characteristics in VAT. ( $A$ and $B$ ) Number of IBA1positive ATM (red) per perilipin-positive adipocytes (green) and crownlike structure (CLS) density were assessed in adipose tissue sections of lean cholecystectomy controls and obese individuals, stratified for liver pathology. Nuclear counterstain was performed using 4,6-diamidin-2phenylindol (DAPI) (blue). Representative images showing a significant increase in the number of ATMs and crown-like structures in obese patients, compared with lean controls. CLS and interstitial ATMs with no contact to CLS are indicated by asterisks and arrows, respectively. (A) Scale bar: 50 $\mu \mathrm{m}$. Flow cytometry was performed and macrophages were identified based on CD45 and CD14 positivity. Within the CD45+ CD14+ gate, the frequency of $\mathrm{CD} 11 \mathrm{c}+$ CD206+ and CD11c+ CCR2+ cells were analyzed and quantified. (C) We showed an increase in the frequency of CD11c+ CD206+ and CD11c+ CCR2+ macrophages in $\mathrm{NASH}$ compared with obese/NAFL and lean cholecystectomy controls. (D) Supernatants of macrophage cultures were assayed for cytokine/chemokine levels after 24 hours. Cytokine and chemokine levels were higher in patients with NASH/ NASH with fibrosis compared with obese/NAFL or lean cholecystectomy controls.
B
C
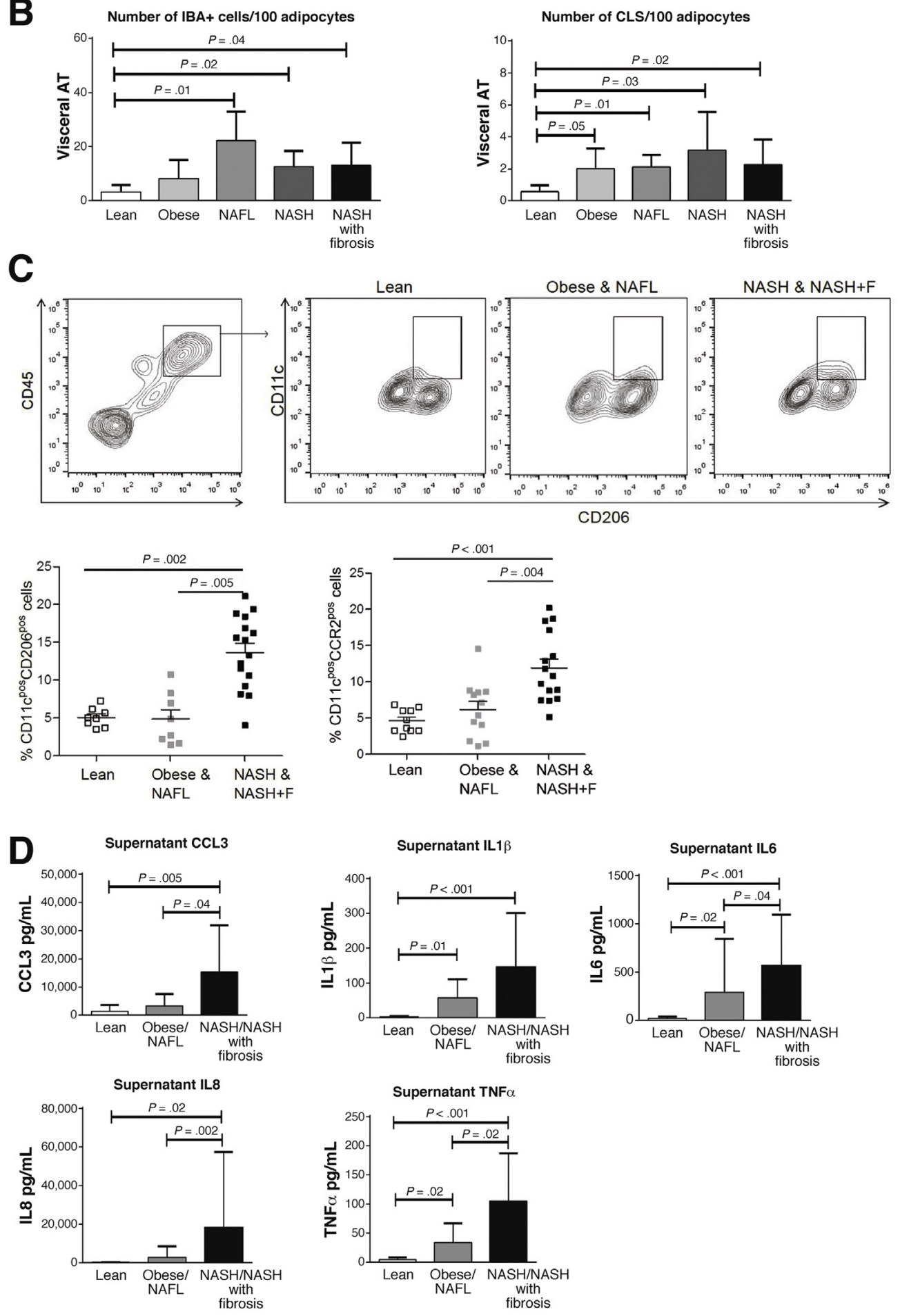
inflammatory cytokines/chemokines by these cells may contribute to the increased cytokine/chemokine levels observed in the circulation in our study and indeed may explain the strong correlation between $\operatorname{TNF} \alpha$, IL8, and CCL3 levels with ALT levels, AST levels, and NAS. TNF $\alpha$ contributes to the development of NASH in human and animal studies. ${ }^{22}$ $\mathrm{Ob} / \mathrm{ob}$ mice show several immunologic abnormalities, including increased production of TNF $\alpha$ by Kupffer cells. ${ }^{23,24}$ IL8 is a potent leukocyte chemoattractant and is secreted by various cells including monocytes and macrophages. IL8 is increased in chronic liver disease, and may contribute to hepatic inflammation by activation of Kupffer cells. ${ }^{24}$ IL8 levels are increased in $\mathrm{NASH}^{25}$ and in obese Hispanic pediatric patients in whom serum IL8 correlated with the hepatic fat fraction measured by magnetic resonance imaging. ${ }^{26}$ CCL3 has been shown to be a mediator of experimental liver fibrosis in mice. ${ }^{27}$ We detected increased CCL3 serum levels in patients with NASH and fibrosis. CCL3 serum levels also correlated strongly with AST and NAS, suggesting that this chemokine might be of particular importance in the development of fibrosis in human beings with NASH.

In the second part of this study we investigated whether gene expression in VAT or SAT with or without additional clinical and biochemical parameters could be used to classify patients into histologic subgroups accurately. We selected 2 sets of genes based on the original microarray analysis performed on VAT and SAT in the training cohort of 35 patients. By using unsupervised hierarchical clustering analysis we could assign individual patients from the training cohort to histologic subgroups with a high degree of accuracy that supported the further use of these selected genes in model building. By using logistic and linear regression, predictive models for VAT and SAT were developed in the training cohort and the performance of these models evaluated in 78 independent patients in the validation cohort. We found that a model obtained using qRT-PCR data from 5 genes in SAT was the most predictive in accurately assigning individuals to the correct histologic subgroup (AUROC, 0.903; sensitivity, 96.97\%; specificity, $72.41 \%$ ). Adding clinical or biochemical information, or the results of cytokine levels, did not improve the performance of this model further.

The significance of SAT inflammation and the highly predictive nature of the gene set in classifying liver histology is of particular importance. Human studies assessing a limited number of genes in VAT have found that increased expression of these proinflammatory genes correlated with liver histology. ${ }^{28,29}$ A recent study showed that macrophages isolated from deep SAT resembled the proinflammatory phenotype of VAT macrophages and was increased significantly in patients with NASH and fibrosis. ${ }^{11}$ Another study also showed that the transcription of proinflammatory genes and macrophage numbers in SAT correlated with hepatic fat content. ${ }^{12}$ Collectively, the published data, together with our results, strongly suggest that SAT also may contribute significantly to NAFL-to-NASH progression.

In the first part of our study we showed the importance of adipose tissue inflammation, which suggested that ATMs are involved in NAFL-to-NASH progression in human beings. To elucidate the phenotype and functional characteristics of ATMs in NAFLD further we isolated these cells from adipose tissue obtained from an additional cohort of bariatric surgery patients stratified according to liver histology, as well as lean cholecystectomy controls.

Recent studies evaluating the role of macrophages in obesity and insulin resistance have confirmed the influx of CCR2 + cells into adipose tissue. ${ }^{19}$ Studies also have shown the presence of 2 dominant macrophage populations at this site: CD11c-CD206+ cells are solitary resident macrophages located at adipocyte junctions that represent the dominant phenotype in lean individuals. CD11c-CD206+ cells secrete IL10, are involved in tissue maintenance and repair, and may facilitate adipogenesis. ${ }^{6,7}$ In contrast, CD11c+CD206+ macrophages increase in obesity and aggregate around necrotic adipocytes in crown-like structures and have been associated with the production of proinflammatory cytokines and insulin resistance. ${ }^{30}$ This subpopulation is capable of metabolizing lipids released by necrotic adipocytes and initiating adaptive immune responses. ${ }^{30}$ In our study using immunofluorescence we confirmed the presence of increased numbers of ATMs in all histologic subgroups compared with lean controls. We showed that the number of ATMs and crownlike structures did not differ between the histologic subgroups. However, we could show, using flow cytometry, an increase in the frequency of CD11c+CD206+ and CCR2+ macrophages in adipose tissue and showed that supernatants of cultured macrophages in NASH had increased levels of cytokines and chemokines, compared with obese/ NAFL or lean cholecystectomy controls. Taken together our data suggest that activated CD11c+CD206+ macrophages are of cardinal importance in NAFL-to-NASH progression.

In this study we stratified patients into 4 distinct histologic subgroups that allowed us to examine differences between the transcriptional profiles of VAT and SAT in severely obese patients with normal liver histology compared with well-delineated subtypes of NAFLD. Future studies, however, should be performed to establish whether our results can be extrapolated to other NAFLD phenotypes with less pronounced obesity.

In conclusion, our analysis suggests that the transcription of proinflammatory genes by visceral and subcutaneous adipose tissue may be a prerequisite for the development of NAFLD in human beings. In addition, our study suggests that CD11c+CD206+ macrophages and the secretion of proinflammatory cytokines/chemokines by ATMs significantly contribute to the pathogenesis of NAFLD, which provides a therapeutic rational for targeting these cells in NASH. Finally, we showed that determining the expression profile of 5 genes in subcutaneous adipose tissue was highly accurate in predicting liver histology. Because of the accessibility of subcutaneous adipose tissue, this approach, if validated in other NASH phenotypes, may be clinically useful in selecting individuals who may benefit from more intense surveillance strategies or therapies in the future. 
Address requests for reprints to: Schalk van der Merwe, MD, MSc, $\mathrm{PhD}$, Department of Internal Medicine, Division of Liver, Gallbladder and Pancreaticobiliary Disorders, University Hospital Gasthuisberg, University of Leuven, Leuven, Belgium. e-mail:

schalk.vandermerwe@uzleuven.be; fax: (32) 16-34-43-87.

\section{References}

1. Gastaldelli A, Cusi K, Pettiti M, et al. Relationship between hepatic/visceral fat and hepatic insulin resistance in nondiabetic and type 2 diabetic subjects. Gastroenterology 2007;133:496-506.

2. van der Poorten $\mathrm{D}$, Milner KL, Hui J, et al. Visceral fat: a key mediator of steatohepatitis in metabolic liver disease. Hepatology 2008;48:449-457.

3. Sun K, Kusminski CM, Scherer PE. Adipose tissue remodelling and obesity. J Clin Invest 2011; 121:2094-2101.

4. Cusi K. Role of obesity and lipotoxicity in the development of nonalcoholic steatohepatitis: pathophysiology and clinical implications. Gastroenterology 2012; 142:711-725.

5. Lumeng CN, Deyoung SM, Saltiel AR. Increased inflammatory properties of adipose tissue macrophages recruited during diet-induced obesity. Diabetes 2007; 56:16-23.

6. Lumeng CN, Bodzin JL, Saltiel AR. Obesity induces a phenotypic switch in adipose tissue macrophage polarization. J Clin Invest 2007;117:175-184.

7. Patsouris D, Li PP, Thapar D, et al. Ablation of CD11Cpositive cells normalizes insulin sensitivity in obese insulin resistant animals. Cell Metab 2008;8:301-309.

8. Kanda H, Tateya S, Tamori Y, et al. MCP-1 contributes to macrophage infiltration into adipose tissue, insulin resistance and hepatic steatosis in obesity. J Clin Invest 2006;116:1494-1505.

9. Kim JY, van de Wall E, Laplante M, et al. Obesity-associated improvements in metabolic profile through expansion of adipose tissue. J Clin Invest 2007; 117:2621-2637.

10. Stanton MC, Chen SC, Jackson JV, et al. Inflammatory signals shift from adipose to liver during high-fat feeding and influence the development of steatohepatitis in mice. J I n flamm (Lond) 2011;8:8-14.

11. Tordjman J, Divoux A, Prifti E, et al. Structural and inflammatory heterogeneity in subcutaneous adipose tissue: relation with liver histopathology in morbid obesity. $J$ Hepatol 2012;56:1152-1158.

12. Munukka E, Pekkala S, Wiklund P, et al. Gut-adipose tissue axis in hepatic fat accumulation in humans. J Hepatol 2014;61:132-138.

13. Brunt EM, Kleiner DE, Wilson LA, et al. Nonalcoholic fatty liver disease (NAFLD) activity score and the histopathologic diagnosis in NAFLD: distinct clinicopathologic meanings. Hepatology 2011;53:810-820.

14. Huang da W, Sherman BT, Lempicki RA. Systematic and integrative analysis of large gene lists using DAVID bioinformatics resources. Nat Protoc 2009; 4:44-57.
15. Franceschini A, Szklarczyk D, Frankild S, et al. STRING v9.1: protein-protein interaction networks, with increased coverage and integration. Nucleic Acids Res 2013;41:D808-D815.

16. Verrijken A, Beckers S, Francque $S$, et al. A gene variant of PNPLA3, but not of APOC3, is associated with histological parameters of NAFLD in an obese population. Obesity 2013;21:2138-2145.

17. Tanwar S, Trembling PM, Guha IN, et al. Validation of terminal peptide of procollagen III for the detection and assessment of nonalcoholic steatohepatitis in patients with nonalcoholic fatty liver disease. Hepatology 2013; 57:103-111.

18. Duval C, Thissen U, Keshtkar S, et al. Adipose tissue dysfunction signals progression of hepatic steatosis towards nonalcoholic steatohepatitis in C57BL/6 mice. Diabetes 2010;59:3181-3191.

19. Weisberg SP, Hunter D, Huber R, et al. CCR2 modulates inflammatory and metabolic effects of high-fat feeding. $\mathrm{J}$ Clin Invest 2006;116:115-124.

20. Moylan CA, Pang $H$, Dellinger $A$, et al. Hepatic gene expression profiles differentiate presymptomatic patients with mild versus severe nonalcoholic fatty liver disease. Hepatology 2014;59:471-482.

21. Marra F, Tacke F. Roles for chemokines in liver disease. Gastroenterology 2014;147:577-594.

22. Wigg AJ, Roberts-Thomson IC, Dymock RB, et al. The role of intestinal bacterial overgrowth, intestinal permeability, endotoxaemia and tumour necrosis factor $\alpha$ in the pathogenesis of non-alcoholic steatohepatitis. Gut 2001; 48:206-211.

23. Weisberg SP, McCann D, Desai $M$, et al. Obesity is associated with macrophage accumulation in adipose tissue. J Clin Invest 2003;112:1796-1808.

24. Abu-Shanab A, Quigley EMM. The role of the gut microbiota in nonalcoholic fatty liver disease. Nat Rev Gastroenterol Hepatol 2010;7:691-701.

25. Du Plessis J, Vanheel $\mathrm{H}$, Janssen CE, et al. Activated intestinal macrophages in patients with cirrhosis release $\mathrm{NO}$ and IL-6 that may disrupt intestinal barrier function. J Hepatol 2013;58:1125-1132.

26. Kim JS, Le KA, Mahurkar S, et al. Influence of elevated liver fat on circulating adipokines and insulin resistance in obese Hispanic adolescents. Pediatr Obes 2012; 7:158164.

27. Heinrichs D, Berres M, Nellen A, et al. The chemokine CCL3 promotes experimental liver fibrosis in mice. Plus One 2013;8:e66106.

28. Estep JM, Baranova A, Hossain N, et al. Expression of cytokine signaling genes in morbidly obese patients with non-alcoholic steatohepatitis and hepatic fibrosis. Obes Surg 2009;19:617-624.

29. Moschen AR, Molnar C, Enrich B, et al. Adipose and liver expression of interleukin (IL)-1 family members in morbid obesity and effects of weight loss. Mol Med 2011; 17:840-845.

30. Wentworth JM, Naselli G, Brown WA, et al. Pro-inflammatory CCD11c+CD206+ adipose tissue macrophages are associated with insulin resistance in human obesity. Diabetes 2010;59:1648-1656. 


\section{Acknowledgments}

The authors wish to acknowledge the contributions of the Vlaams Instituut voor Biotechnologie (VIB-Nucleomics Core), University of Leuven Belgium, for excellent assistance in the microarray experiments, and Sigri Beckers and Doreen Zegers (Department of Medical Genetics, University of Antwerp, Belgium) for assistance with PNPLA3 genotyping. The authors also wish to acknowledge the contributions of Professor E. Van Marck, and Dr M. Ruppert (University of Antwerp) for the analysis of the histologic specimens and for the collection of liver and adipose tissue samples during surgery, respectively. The authors also wish to acknowledge Professor Gert de Hertogh, Professor Tania Roskams, and Kathleen van den Eynde (Department of Pathology, University of Leuven) for the preparation of the adipose tissue samples for immunohistochemistry. The authors would like to thank Professor Johan Fevery for valuable comments and suggestions during the first draft of the manuscript.

\section{Conflicts of interest}

The authors disclose no conflicts.

\section{Funding}

Schalk van der Merwe, Chantal Mathieu, Frederik Nevens, David Cassiman, and Sven Francque are recipients of the Flanders fund for scientific research (FWO klinisch mandaat), and Hannelie Korf is a recipient of the FWO postdoctoral mandate. Research at the Department of Endocrinology, Diabetology and Metabolism and the Department of Gastroenterology and Hepatology of the Antwerp University Hospital (Belgium) was supported by the European Union: FP6 (HEPADIP contract LSHM-CT-2005-018734) and FP7-HEALTH (RESOLVE no. 305707). Supported by a fellowship from the South African Gastroenterology Association and a scholarship from the European Association for the Study of the Liver (J.d.P.). This research also was supported by a research grant from the Gastro foundation of South Africa. The authors specifically acknowledge the support of Dr. Chris Kassianides. Also funded in part by a grant from the Deutsche Forschungsgemeinschaft DFG-SFB 1052/1: Obesity Mechanisms (projects A04) and by the Helmholtz Alliance Imaging and Curing Environmental Metabolic Disease through the Initiative and Networking Fund of the Helmholtz Association (M.G.). 


\section{Supplementary Materials and Methods}

\section{Anthropometric Measurements}

All measurements were performed in the morning, with patients in fasting conditions and undressed. Height was measured to the nearest $0.5 \mathrm{~cm}$ and body weight was measured with a digital scale to the nearest $0.2 \mathrm{~kg}$. BMI was calculated as weight (in kilograms) over height (in meters) squared. Waist circumference was measured at the midlevel between the lower rib margin and the iliac crest. Hip circumference was measured at the level of the trochanter major. The waist-to-hip ratio was calculated by dividing the waist circumference by the hip circumference. The body composition of the patients from Antwerp was determined by bio-impedance analysis as described by Lukaski et al, ${ }^{1}$ and fat mass percentage was calculated using the formula of Deurenberg et al. ${ }^{2}$ For all patients from South Africa, the percentage of body fat was measured by means of dualenergy x-ray absorptiometry with a GE Lunar Prodigy scanner (serial number 77060GA, Buckinghamshire, UK) for patients who weighed more than $180 \mathrm{~kg}$.

\section{Histology}

Histologic scoring was performed by an expert pathologists blinded to clinical information. Steatosis and other features of NASH were scored semiquantitatively according to the criteria cited in Supplementary Table 1. To quantify the severity of disease the unweighted sum of scores of steatosis, hepatocyte ballooning, and lobular inflammation was determined according to the NASH Clinical Research Network Scoring System.

\section{RNA Extraction, cDNA Synthesis, and Quantitative RT-PCR}

Adipose tissue was homogenized with TRIzol (Invitrogen Life Technologies, Carlsbad, CA) and RNA was isolated by the RNeasy Kit (Qiagen). RNA quality and quantity was determined by a NanoDrop spectrophotometer (NanoDrop Technologies, Centreville, DE) and an Agilent 2100 BioAnalyzer (Agilent, Palo Alto, CA), respectively. cDNA was synthesized from $2.5 \mu \mathrm{g}$ of RNA using SuperScript II reverse transcriptase and random hexamer primers (Invitrogen Life Technologies). Quantitative RT-PCR was performed with TaqMan Universal PCR Master Mixture (Applied Biosystems, Foster City, CA) and Assays-on-Demand Gene Expression products (Applied Biosystems). Real-time PCR amplification and data analysis were performed using the A7500 Fast Real-Time PCR System (Applied Biosystems). Each sample was assayed in duplicate. The $\Delta \Delta \mathrm{Ct}$ method was used to determine the relative gene expression levels with $\beta 2$ microglobulin as the reference gene. Primer details are listed in Supplementary Table 2.

\section{Microarray and Data Processing}

A total of $100 \mathrm{ng}$ per sample of total RNA spiked with bacterial RNA transcript-positive controls (Affymetrix) was amplified and labeled using the GeneChip $3^{\prime}$ IVT express kit
(Affymetrix). All steps were performed according to the manufacturer's protocol (Affymetrix). A mixture of purified and fragmented biotinylated aRNA and hybridization controls (Affymetrix) were hybridized on Affymetrix Primeview arrays followed by staining and washing in a GeneChip fluidics station 450 (Affymetrix) according to the manufacturer's procedures. To assess the raw probe signal intensities, chips were scanned using a GeneChip scanner 3000 (Affymetrix). Analysis of the microarray data was performed in the R programming environment, in conjunction with the packages developed within the Bioconductor project. ${ }^{4}$ The analysis was based on the RMA expression levels of the probe sets, computed with the package xps (version 1.16.0) (Christian Stratowa, Vienna, Austria). Differential expression was assessed via the moderated $t$-statistic, described by Smyth et al, ${ }^{5,6}$ and implemented in the limma package (version 3.12.1) (Gordon Smyth et al http:// bioinf.wehi.edu.au/limma). To control the false-discovery rate, multiple testing corrections were performed. ${ }^{7}$ Microarray results were confirmed by performing qRT-PCR on a limited number of genes (Supplementary Table 3).

\section{Pathway Analysis}

Gene enrichment and pathway analysis was performed with the internet-based DAVID Bioinformatics Resource 6.7 program suite (http://david.abcc.ncifcrf.gov/). ${ }^{8}$ Analysis was performed on the list of up-regulated and downregulated genes with a fold-change of $\geq 1$ or $\leq-1$. Kyoto Encyclopedia of Genes and Genomes pathways and the gene ontology terms of cellular component, molecular function, and biological process in DAVID were used to categorize enriched biological themes.

To explore functional interactions or partnerships between the genes we loaded them into the STRING 9.1 program (http://string-db.org/). ${ }^{9}$ This program weights and integrates information from numerous sources, including experimental repositories, computational prediction methods, and public text collections, thus acting as a metadatabase that maps all interaction evidence onto a common set of genomes and proteins. In addition to a graphic representation of the predicted interactions, the program generates a list of the most relevant gene-to-gene interactions. The complexity of the interactions can be visualized where a blue line represents a reported relationship between the 2 genes and the thickness of the line represents the confidence (more literature results show a thicker line).

\section{Cytokine and Chemokine Measurements}

Blood was collected aseptically from a peripheral vein before surgery, centrifuged, and stored at $-80^{\circ} \mathrm{C}$ until further analysis. In a subgroup of 78 patients Custom Meso Scale Discovery V-plex assays were used to determine plasma cytokines (IL1 $\beta, 6,8,10$, and $\operatorname{TNF} \alpha$ ) and chemokines (monocyte chemotactic protein 1/CCL2), monocyte chemoattractant protein 4/CCL13, macrophage-derived chemokines, and macrophage inflammatory protein $1 \alpha /$ CCL3 levels. All measurements were performed in duplicate (Supplementary Table 4). 


\section{Flow Cytometric Analysis and Culture of VAT Macrophages}

Adipose tissue samples $(1 \mathrm{~g})$ were sliced into small pieces using sterile scalpels before dissociation of the tissue with a GentleMACS homogenizer (Miltenyi Biotec, Bergisch Gladbach, Germany) and digestion with type IV collagenase $\left(15 \mathrm{~min}\right.$ at $\left.37^{\circ} \mathrm{C}\right)(4 \mathrm{mg} / \mathrm{mL})$ (Sigma-Aldrich, St. Louis, MO). The homogenization and digestion of the samples were repeated and cell clumps were removed by passing the solution through a $70-\mu \mathrm{m}$ cell strainer. The single-cell suspensions then were subjected to a series of centrifugation and wash steps. Finally, the cells were counted and the leukocyte populations were analyzed by flow cytometry. All staining procedures were performed in $\mathrm{Ca} 2+-$ and $\mathrm{Mg} 2+-$ free phosphate-buffered saline containing $2 \mathrm{mmol} / \mathrm{L}$ EDTA and $0.1 \%$ bovine serum albumin.

Cells were preincubated with Fc block to minimize nonspecific binding. Cells $(2 \times 105)$ were labeled directly with the following conjugated antibodies: CD45, CD14, HLADR, CD33, CD11b, CD11c, TREM-1, CCR2, and matching isotype controls. All antibodies were obtained from eBioscience, and CD206, which was from BD Biosciences. Data acquisition was performed on a Gallios flow cytometer (Beckman Coulter) and the data were analyzed using FlowJo V.10 software (www.flowjo.com/download/). Leukocytes were identified based on side scatter and CD45 and adipose tissue macrophages subsequently were analyzed within the leukocyte population based on CD14 and CD45 expression. Additional macrophage markers (CD206 and CD11c) were analyzed within this population.

In addition, the isolated cells from VAT tissue were resuspended in RPMI medium containing $10 \%$ heatinactivated fetal calf serum, $100 \mathrm{U} / \mathrm{mL}$ penicillin, $100 \mathrm{mg} /$ $\mathrm{mL}$ streptomycin, and $50 \mathrm{mmol} / \mathrm{L} \quad \beta$-mercaptoethanol cultured. Cells $\left(2 \times 10^{5}\right)$ were plated in a 96-well tissue culture plate and incubated at $37^{\circ} \mathrm{C}$ for 1 hour before washing away the nonadherent cells. The latter procedure routinely resulted in more than $90 \%$ macrophage enrichment as assessed by CD45+CD14+ positivity (not shown). After an additional 24-hour incubation at $37^{\circ} \mathrm{C}$ the culture media were collected, centrifuged, and stored at $-20^{\circ} \mathrm{C}$. Custom Meso Scale Discovery V-plex assays were used to determine macrophage-derived cytokine and chemokine production (Supplementary Table 5).

\section{Immunofluorescence Staining of AT Sections}

Human samples from visceral AT were fixed immediately in 6\% PFA, dehydrated through ascending grades of ethanol, and embedded in paraffin. AT sections (7$\mu$ m-thick) were deparaffinized in xylene and rehydrated through descending grades of ethanol to water. Antigen retrieval was performed in Tris/EDTA buffer at $\mathrm{pH} 9.5$ twice for 5 minutes at $95^{\circ} \mathrm{C}$. Sections were rinsed in phosphatebuffered saline supplemented with $0.3 \%$ Triton-X (SigmaAldrich, St Lois, Missouri, USA) 3 times for 5 minutes. Unspecific binding sites were blocked using $1 \%$ bovine serum albumin in phosphate-buffered saline supplemented with $0.3 \%$ Triton-X for 30 minutes at room temperature.
Subsequently, rabbit anti-Iba1 (1:500, ordering number 019-19741; Wako, Richmond, VA) and goat anti-perilipin A (1:200, ab61682; Abcam) diluted in phosphate-buffered saline supplemented with $0.3 \%$ Triton-X with $1 \%$ bovine serum albumin were applied and incubated overnight at $4^{\circ} \mathrm{C}$. The next day, sections were buffer-rinsed and incubated with donkey anti-goat Alexa 488 and donkey antirabbit Alexa 568 (diluted at 1:200 in phosphate-buffered saline supplemented with $0.3 \%$ Triton- $X$ with $1 \%$ bovine serum albumin; both from Invitrogen, Karlsruhe, Germany) for 1 hour at room temperature. Autofluorescence of the tissue was quenched by using prewarmed $0.3 \%$ sudan black for 2 minutes. Nuclear counterstain was performed by using 4,6-diamidin-2-phenylindol $\quad(1: 10,000$ in phosphatebuffered saline) for 5 minutes, followed by 3 buffer rinses. Finally, sections were embedded with Dako immunofluorescence mounting medium (DAKO, Glostrup, Denmark).

\section{Analysis of AT Sections}

For morphologic analysis, the pan-macrophage marker Iba1 and the adipocyte marker perilipin A were stained as described earlier. Three representative images were taken randomly per AT sample at a low magnification $(100 \times)$ with identical settings and a minimum distance of $50 \mu \mathrm{m}$ from each other. The number of adipocytes per section, adipocyte area, adipocyte diameter, and number of ATMs per section were quantified in an unbiased fashion using cellSens Software (Olympus, Hamburg, Germany). Initially, threshold and pixel area filter settings of cellSens software were adjusted manually to either adipocytes or ATMs and remained unchanged for analyses of all sections. Crown-like structures were characterized as a minimum of 3 ATMs surrounding a perilipin-negative adipocyte and manually counted in each section, as described earlier. ${ }^{10,11}$ Image acquisition and analyses were performed blinded to the investigator.

\section{Supplementary Results}

\section{Patient Characteristics}

Additional patient characteristics are shown in Supplementary Tables 6-8.

\section{Expression Changes in the Liver}

Microarray analysis was performed on liver tissue corresponding to the patients from whom we also had investigated the fat in this study: 15 morbidly obese patients ( 6 obese, 4 NAFL, and 5 NASH). By using the criteria of $\Delta$ gene expression $2 \log$ value greater than +1 or $<-1$, we identified nearly 150 genes that subsequently were used for pathway analysis. Between obese and NAFL, there were 37 genes up-regulated and 27 genes that were down-regulated. Between NAFL and NASH we found 60 genes up-regulated and 38 genes that were down-regulated. Supplementary Figure $1 A$ shows the interactions between the differentially expressed genes as determined by String9.1. In addition, 5 Kyoto Encyclopedia of Genes and Genomes pathways 
were identified (Supplementary Figure $1 B$ ). In the liver, changes in gene expression mostly were related to metabolic processes, cholesterol biosynthesis, and so forth, and not inflammation. The expression changes in liver metabolism and fatty acid biosynthesis were similar to a recently published study by Moylan et al. ${ }^{12}$ They assessed gene expression profiles in liver tissue using microarray in individuals at "low" and "high" risk for NASH.

\section{Detailed Description of Gene Expression in VAT and SAT}

A general overview of the microarray results are presented in Supplementary Table 9. In VAT we observed an increase in the number of differentially expressed genes in the more advanced stages. This was less prominent in SAT. Pathway analysis in visceral fat showed that the largest number of pathways that are different can be found between NAFL and NASH. Two clusters of pathways appear to emerge centered around cancer and cytokine/chemokines, however, many of the genes were overlapping between these pathways. The pathways that change between NASH and fibrosis are considerably less, only 3 pathways were identified but with a higher statistical relevance because the focus was on cytokine and chemokine signaling. The gene ontology biological processes that were withheld by DAVID underline the results of the pathway analysis (Supplementary Tables 9-11).

The number of genes that were significantly expressed differentially (2logratio $>1$ or $<-1$ ) according to the microarray analysis between the histologic groups for VAT and SAT. (Probes: number of probes identified to be expressed differentially by microarray; genes: number of different genes corresponding to the identified probes; David6.7: number of genes annotated by the DAVID Bioinformatics Resource 6.7 program $^{8}$; Kyoto Encyclopedia of Genes and Genomes: number of Kyoto Encyclopedia of Genes and Genomes pathways identified by David6.7; gene ontology Biological Processes: number of biological processes identified by David6.7 with a $P$ value less than .05 after Bonferroni correction).

\section{Correlation of Expression of Cytokine and Chemokine Genes in SAT With That in VAT}

For the 113 patients, gene expression was determined by qRT-PCR in visceral and subcutaneous adipose tissue and the Pearson product moment correlation was calculated. The $\mathrm{r}$ provides the correlation coefficient and the $P$ values provides the probability. A $P$ value less than .05 was considered statistically significant.

\section{Bland-Altman Analysis}

This was investigated further by Bland-Altman analysis. For 6 genes the expression was near-equal in both compartments with a significant Pearson correlation as well (CCL2, IL1RN, IL8, SERPINE1, JUN, and RELN). For CCL3, the level of expression was near-equal but without a significant Pearson correlation. For CCL21 and GADD45B we found a lower expression in the visceral fat than in the subcutaneous fat (Supplementary Figure 2).

\section{Model Development and Evaluation}

For all of the selected genes we determined their expression by qRT-PCR. Subsequently, we performed mathematic model building for the training set to obtain an equation that accurately can predict liver histology from a limited number of parameters (MedCalc Software bvba; http://www.medcalc.org; 2014). As input for our models we used 18 biochemical and clinical parameters in combination with the qRT-PCR data for the 35 patients.

Two types of regression models were investigated for this study: multiple linear regression and multiple logistic regression. In linear regression, data are modeled using linear predictor functions, and unknown model parameters (liver histology: obese control, NAFL, NASH, NASH with fibrosis) are estimated from the data (gene expression, biochemical parameters). Logistic regression, or logit regression, is a type of probabilistic statistical classification model. It is used to predict a binary response (here liver histology control/NAFL $=0$ and liver histology NASH/ NASH with fibrosis $=1$ ) from 1 or more predictor variables (gene expression, biochemical parameters). Both are used in estimating empiric values of the parameters in a qualitative response model. ${ }^{13-15}$ Selection was performed using backward and forward analysis to obtain the optimal predictive formula (model) using weighted contribution of the variables. Performance of the models was investigated in the independent validation cohort of 78 patients. Blinded for the histologic classification, the regression gene score was calculated and the patients were classified using the criteria developed for the corresponding training cohort. AUROC analysis was performed to assess the sensitivity and specificity.

\section{The Performance of Serum Markers in Gene- Based Obesity Classification}

A total of 9 cytokines and chemokines were determined in the serum of the 76 patients. By using the groups of patients for the training and validation cohorts as was used previously for model building, we had 16 patients in the training group and 60 patients in the validation group. We found that addition of 1 or more cytokine or chemokine parameters to the model did not improve the performance of either one of the models (VAT or SAT) that we had computed by regression building (data not shown).

\section{Performance of Serum Marker N-Terminal Peptide of Procollagen III for Classification of Morbidly Obese Patients}

Recently, the serum marker N-terminal peptide of procollagen III was proposed as a specific and sensitive marker to predict liver histology. ${ }^{16}$ To evaluate the performance of this marker in comparison with our models to predict liver histology we determined the procollagen III $\mathrm{N}$-terminal propeptide by enzyme-linked immunosorbent assay in a subset of 76 of our patients from whom serum samples also were available. This subset of 76 patients was 
fully representative of the original 113 patients in clinical and biochemical characteristics and class distribution. We found that a large portion (43.75\%) of the patients had undetectable levels of procollagen III N-terminal propeptide, in the (obese control or NAFL) group as well as in the (NASH or fibrosis) group. Statistically, procollagen III Nterminal propeptide could discriminate between (obese control or NAFL) and (NASH or fibrosis). AUROC analysis showed the following results: AUC of $0.692(P=.001)$, sensitivity of $69.23 \%$, and specificity of $67.57 \%$, which were below that of both the SAT as well as the VAT (Supplementary Figure 3A).

\section{Performance of the Genetic Marker PNPLA3 Variant rs738409 for Classification of Morbidly Obese Patients}

In addition, mutation analysis of the genetic marker PNPLA3_rs738409 was performed to investigate whether it could discriminate between obese/NAFL vs NASH/NASH with fibrosis. ${ }^{17}$ To evaluate the performance of this marker in comparison with our models to predict liver histology we performed genotyping in a subset of 76 of our patients, fully representative for the original 113 patients in clinical and biochemical characteristics and class distribution. If we compared wild type vs mutation (heterozygote or homozygote) we found statistically more mutations in the NASH or fibrosis groups than in the obese control or NAFL groups $(P=.003)$. AUROC analysis showed the following results: AUROC of $0.691(P=.001)$, sensitivity of $56.8 \%$, and specificity of 77.8\% (Supplementary Figure 3B).

\section{References}

1. Lukaski HC, Johnson PE, Bolonchuk WW, et al. Assessment of fat-free mass using bioelectrical impedance measurements of the human body. Am J Clin Nutr 1985;41:810-817.

2. Deurenberg P, Weststrate JA, Haustvast JGAJ. Changes in fat-free mass during weight loss measured by bioelectrical impedance and by densitometry. Am J Clin Nutr 1989;49:33-36.

3. Kleiner DE, Brunt EM, Van Natta M, et al. Design and validation of a histological scoring system for nonalcoholic fatty liver. Hepatology 2005;41:1313-1321.

4. Gentleman RC, Carey VJ, Bates DM, et al. Bioconductor: open software development for computational biology and bioinformatics. http://www.bioconductor.org. Genome Biol 2004;5:R80.

5. Smyth GK. Linear models and empirical Bayes methods for assessing differential expression in microarray experiments. Stat Appl Genet Mol Biol 2004;3:Article 3.

6. Smyth GK, Michaud J, Scott H. The use of within-array replicate spots for assessing differential expression in microarray experiments. Bioinformatics 2005; 21:2067-2075.

7. Benjamini $Y$, Hochberg Y. Controlling the false discovery rate: a practical and powerful approach to multiple testing. J R Stat Soc Ser B 1995;57:289-300.

8. Huang da W, Sherman BT, Lempicki RA. Systematic and integrative analysis of large gene lists using DAVID bioinformatics resources. Nat Protoc 2009;4:44-57.

9. Franceschini A, Szklarczyk D, Frankild S, et al. STRING v9.1: protein-protein interaction networks, with increased coverage and integration. Nucleic Acids Res 2013; 41:D808-D815.

10. Cinti S, Mitchell G, Barbatelli G, et al. Adipocyte death defines macrophage localization and function in adipose tissue of obese mice and humans. J Lipid Res 2005; 46:2347-2355.

11. Wentworth JM, Naselli G, Brown WA, et al. Proinflammatory CD11c+CD206+ adipose tissue macrophages are associated with insulin resistance in human obesity. Diabetes 2010;59:1648-1656.

12. Moylan CA, Herbert $P$, Dellinger $A$, et al. Hepatic gene expression profiles differentiate pre-symptomatic patients with mild versus severe nonalcoholic fatty liver disease. Hepatology 2014;59:471-482.

13. Harrell FE. Regression modeling strategies: with applications to linear models, logistic regression, and survival analysis. New York, NY: Springer, 2001.

14. Nick TG, Campbell KM. Logistic regression. Methods Mol Biol 2007;404:273-301.

15. Eberly LE. Multiple linear regression methods. Mol Biol 2007;404:165-187.

16. Tanwar S, Trembling PM, Guha IN, et al. Validation of terminal peptide of procollagen III for the detection and assessment of nonalcoholic steatohepatitis in patients with nonalcoholic fatty liver disease. Hepatology 2013; 57:103-111.

17. Verrijken A, Beckers S, Francque $S$, et al. A gene variant of PNPLA3, but not of APOC3, is associated with histological parameters of NAFLD in an obese population. Obesity 2013;21:2138. 


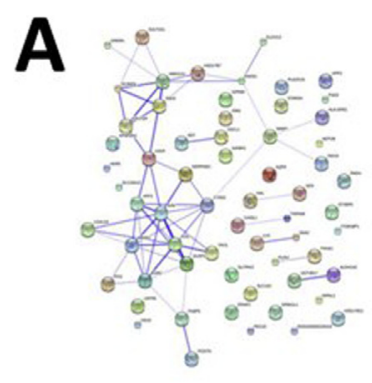

Obese to NAFL

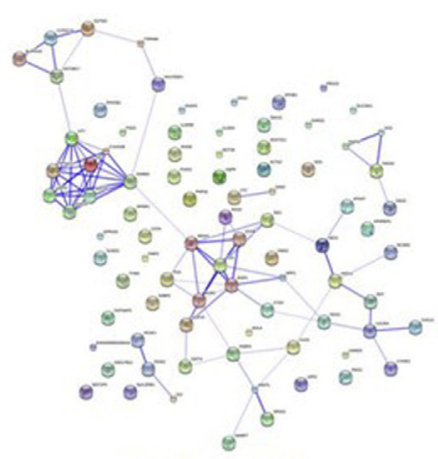

NAFL to NASH

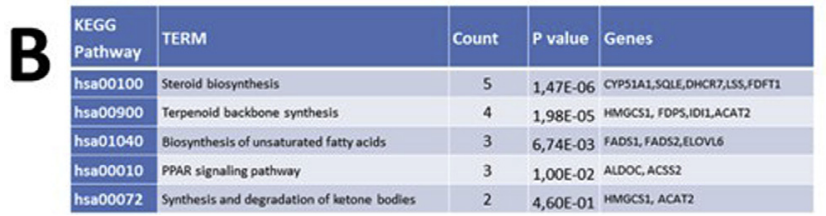

Supplementary Figure 1. (A) Representation of the complexity of the molecular interaction for the differentially expressed genes in liver tissue of morbidly obese patients between obese control and NAFL and between NAFL and NASH. (B) Pathways in the liver that were identified by the DAVID Bioinformatics Resource 6.7 program as being important were related to steroid and free fatty acid biosynthesis. KEGG, Kyoto Encyclopedia of Genes and Genomes. 

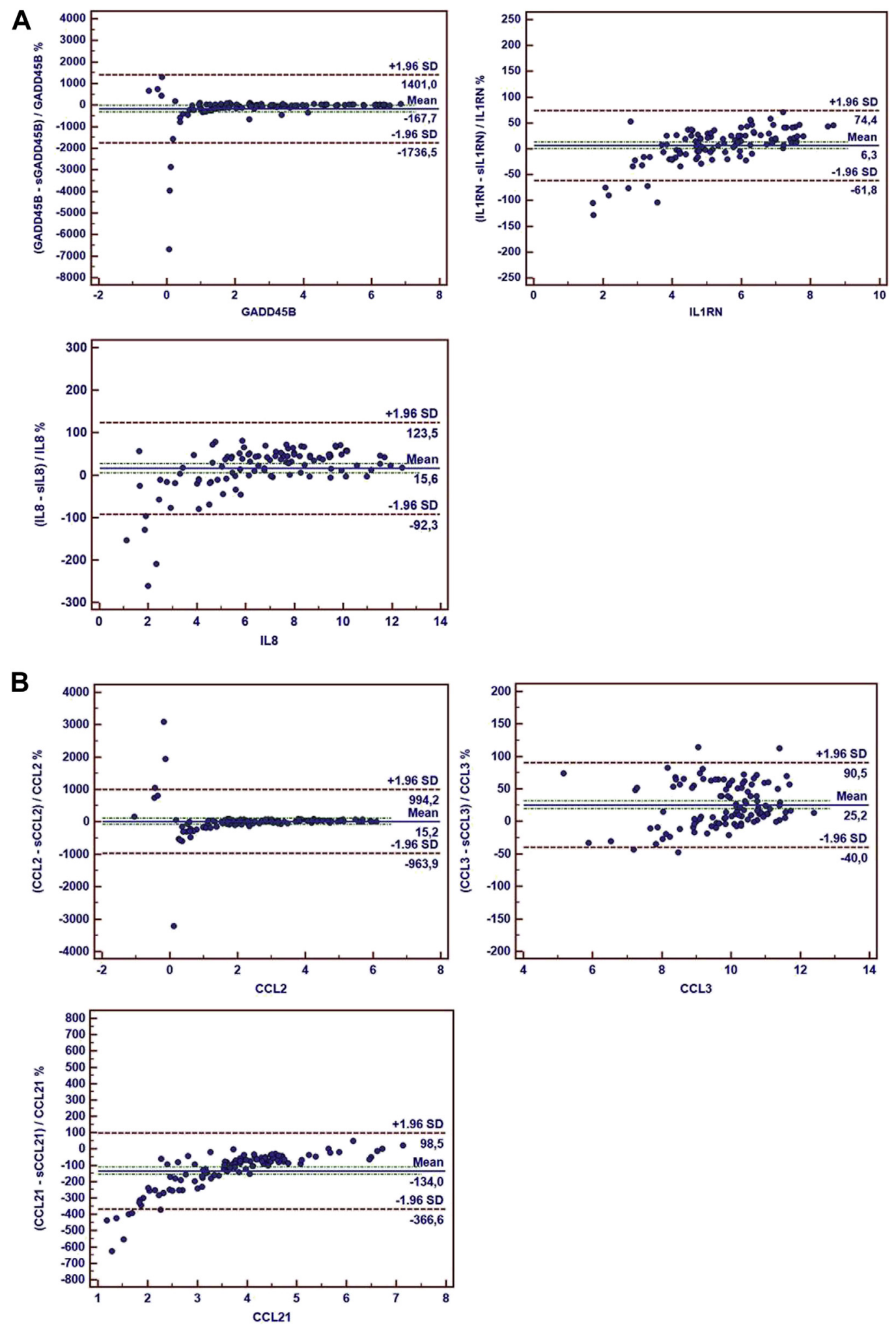

Supplementary Figure 2. Bland-Altman plot VAT gene expression vs SAT gene expression. In addition to Pearson correlation, Bland-Altman plots were constructed. We compared expression in visceral adipose tissue gene expression (GADD45B = expression in VAT) with subcutaneous adipose tissue gene expression (indicated with small "s" in sGADD45B and as for the other genes). The relative difference for each gene is shown (GADD45B - sGADD45B)/GADD45B \%. (A) GADD45B, IL1RN, and IL8. (B) CCL2, CCL3, and CCL21. (C) JUN, RELN, and SERPINE. 

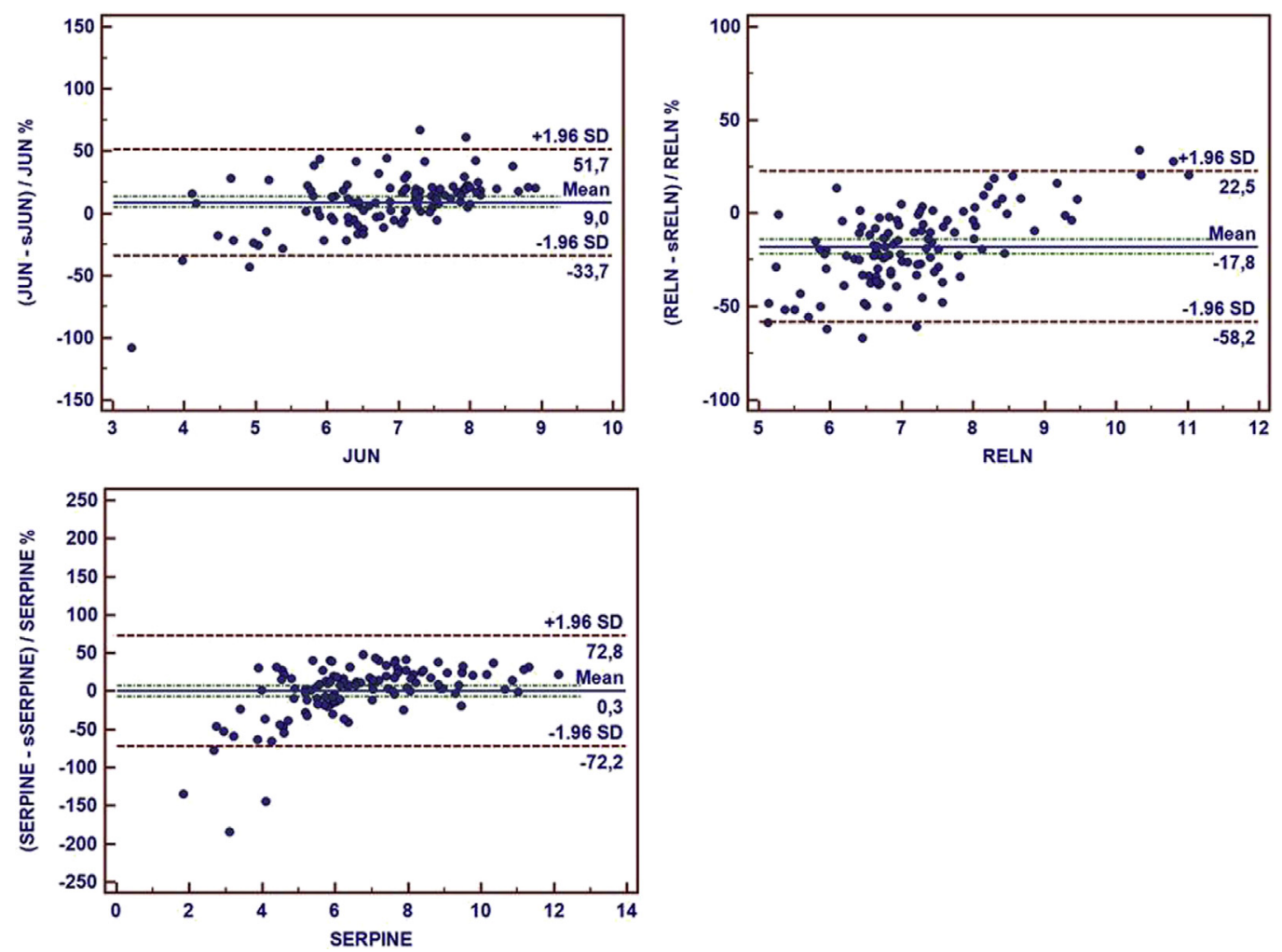

Supplementary Figure 2. (continued).
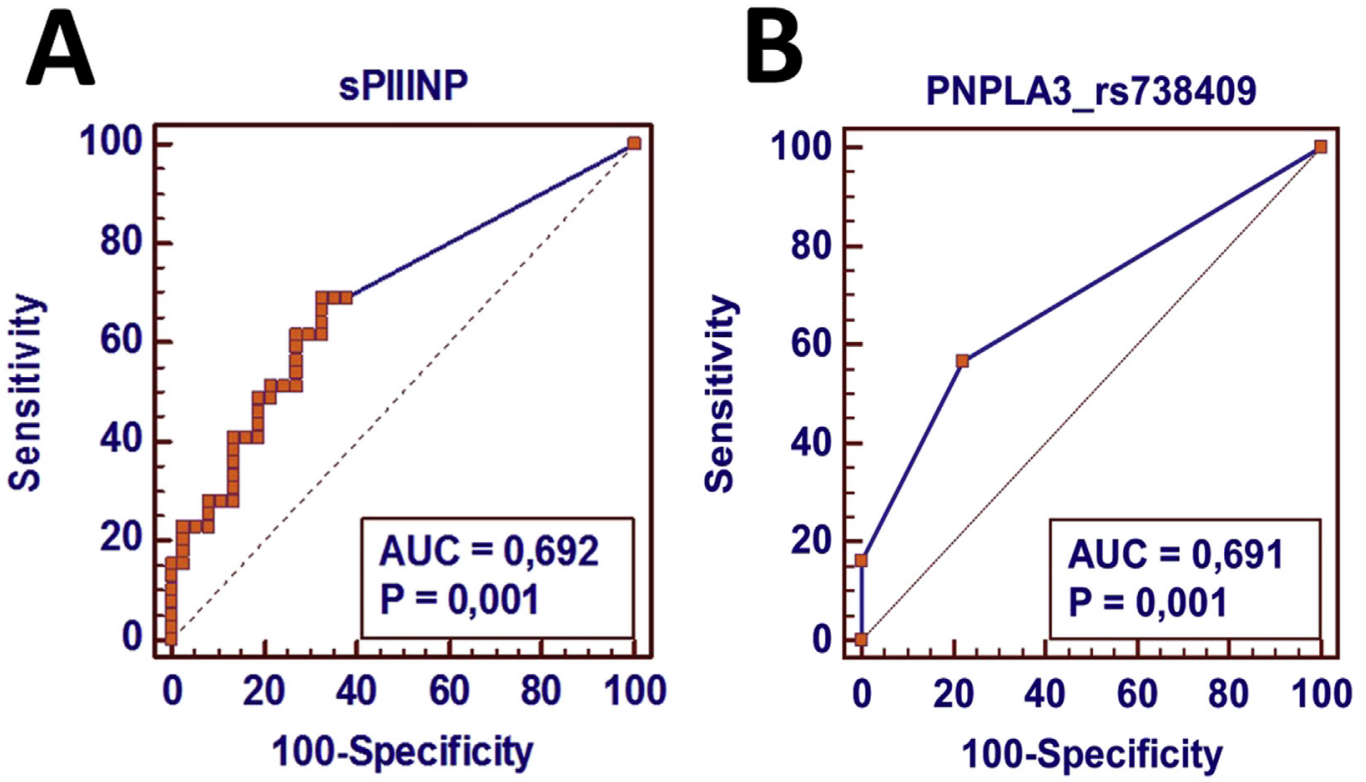

Supplementary Figure 3. Evaluation of serum procollagen III N-terminal propeptide (sPIIINP) and genetic marker PNPLA3 variant rs738409 as a marker for NASH. (A) From 76 patients, equally distributed over the 4 histologic classes, PIIINP was determined by an enzyme-linked immunosorbent assay kit (SEA573Hu; Cloud-Clone Corp). Receiver operating characteristics curve analysis was performed to classify liver histology into (obese control + NAFL) or (NASH + fibrosis). (B) Genotyping for PNPLA3 variant rs738409 was performed. If we compare wild type vs having a mutation (heterozygote or homozygote), we found statistically more mutations in the NASH or fibrosis groups than in the obese control or NAFL groups. 
Supplementary Table 1.NAS Scoring System

\begin{tabular}{ll}
\hline Fibrosis stage & $=$ None \\
1 & $=$ Perisinusoidal or periportal \\
2 & $=$ Perisinusoidal and portal/periportal \\
3 & $=$ Bridging fibrosis \\
4 & $=$ Cirrhosis \\
0 & $=<5 \%$ \\
1 & $=5 \%-33 \%$ \\
2 & $=>33 \%-66 \%$ \\
3 & $=>66 \%$ \\
Steatosis grade & $=$ None \\
1 & $=$ Few balloon cells \\
2 & $=$ Many cells/prominent ballooning \\
0 & $=$ No foci \\
1 & $=<2$ foci per $200 \times$ field \\
2 & $=2-4$ foci per $200 \times$ field \\
3 & $=>4$ foci per $200 \times$ field \\
Lobular inflammation $\quad$ &
\end{tabular}

Supplementary Table 2. Primers Used in This Study

\begin{tabular}{|c|c|c|c|}
\hline Symbol & Gene name & $\begin{array}{c}\text { Chromosomal } \\
\text { location }\end{array}$ & Product reference \\
\hline$B 2 M$ & $\beta 2$-microglobulin & 15 & Hs99999907_m1 \\
\hline CCL2 & Chemokine (C-C motif) ligand 2 & 17 & Hs00234140_m1 \\
\hline CCL21 & Chemokine (C-C motif) ligand 21 & 9 & Hs00989654_g1 \\
\hline CCL3 & Chemokine (C-C motif) ligand 3 & 17 & Hs04194942_s1 \\
\hline DMRT2 & Doublesex and mab-3-related transcription factor 2 & 9 & Hs00246364_m1 \\
\hline GADD45B & Growth arrest and DNA-damage-inducible, $\beta$ & 19 & Hs04188837_g1 \\
\hline IL1RN & Interleukin 1-receptor antagonist & 2 & Hs00893626_m1 \\
\hline IL8 & Interleukin 8 & 4 & Hs00174103_m1 \\
\hline JUN & Jun proto-oncogene & 1 & Hs01103582_s1 \\
\hline$R E L N$ & Reelin & 7 & Hs01022646_m1 \\
\hline SERPINE1 & Serpin peptidase inhibitor, clade $\mathrm{E}$ & 7 & Hs01126606_m1 \\
\hline ZNF880 & Zinc finger protein 880 & 19 & Hs04190223_m1 \\
\hline
\end{tabular}

NOTE. This study used the Assays-on-Demand Gene Expression Primer/Probe Set (Applied Biosystems). 
Supplementary Table 3. Correlation Between Microarray and qRT-PCR $(n=35)$

\begin{tabular}{|c|c|c|c|c|c|}
\hline \multicolumn{3}{|c|}{ Visceral adipose tissue } & \multicolumn{3}{|c|}{ Subcutaneous adipose tissue } \\
\hline Gene & Correlation coefficient & $P$ value & Gene & Correlation coefficient & $P$ value \\
\hline CCL21 & $r=0.874$ & $<.001$ & CCL21 & $r=0.706$ & $<.001$ \\
\hline CCL3 & $r=0.398$ & .004 & IL1RN & $r=0.775$ & $<.001$ \\
\hline ILIRN & $r=0.917$ & $<.001$ & IL8 & $r=0.964$ & $<.001$ \\
\hline$R E L N$ & $r=0.726$ & $<.001$ & SERPINE & $r=0.893$ & $<.001$ \\
\hline \multirow[t]{2}{*}{ SERPINE } & $r=0.745$ & $<.001$ & $D M R T 2$ & $r=0.805$ & $<.001$ \\
\hline & & & ZNF880 & $r=0.712$ & $<.001$ \\
\hline
\end{tabular}

Supplementary Table 4. Lower Limit of Detection for Each Protein Assay According to the Manufacturers

\begin{tabular}{lcc}
\hline & $\begin{array}{c}\text { Lower limit of } \\
\text { detection, } \\
p g / m L\end{array}$ & Assay \\
\hline Procollagen III & $<27.2$ & $\begin{array}{c}\text { SEA573Hu } \\
\text { N-terminal propeptide }\end{array}$ \\
IL1 $\beta$ & 0.04 & (Cloud-Clone Corp) \\
IL6 & 0.06 & MSD V-PLEX \\
IL8 & 0.08 & MSD V-PLEX \\
IL10 & 0.03 & MSD V-PLEX \\
MCP-1 & 0.09 & MSD V-PLEX \\
MCP-4 & 1.69 & MSD V-PLEX \\
MDC & 1.22 & MSD V-PLEX \\
MIP-1 $\alpha$ & 3.02 & MSD V-PLEX \\
& & \\
\hline MCP-1, macred & & \\
\hline
\end{tabular}

MCP-1, macrophage chemotactic protein 1; MCP-4, macrophage chemoattractant protein 4; MDC, macrophage-derived chemokine; MIP, macrophage inflammatory protein. 
Supplementary Table 5. Antibodies Used for Flow Cytometry

\begin{tabular}{ll}
\hline \multicolumn{1}{c}{ Antibody } & Company \\
\hline CD14-APC-eFluor 780 & eBioscience \\
CD45-PerCPCy 5.5 & eBioscience \\
CD33-PECy7 & eBioscience \\
CD11b-APC & eBioscience \\
HLA-DR-FITC & eBioscience \\
CD11C-eFluor450 & eBioscience \\
CCR2-PE & R\&D Systems \\
TREM-1-PE & R\&D Systems \\
CD206-PE & BD Bioscience \\
TLR2-PE & eBioscience \\
TLR4-PE & eBioscience \\
& \\
\hline
\end{tabular}

Supplementary Table 6. Clinical Characteristics of Patients in the Training and Validation Sets

\begin{tabular}{|c|c|c|c|c|c|c|c|}
\hline \multirow[b]{2}{*}{ Characteristic } & \multicolumn{3}{|c|}{ Training cohort $(n=35)$} & \multicolumn{4}{|c|}{ Validation cohort $(\mathrm{n}=78)$} \\
\hline & Median & $25 \%$ & $75 \%$ & Median & $25 \%$ & $75 \%$ & $P$ value \\
\hline Sex, \% male & $17 \%$ & & & $31 \%$ & & & NS \\
\hline Number of patients per group, I-II-III-IV & $11-9-9-6$ & & & $17-16-32-13$ & & & NS \\
\hline Age, $y$ & 43 & 36 & 49 & 43 & 34 & 49 & NS \\
\hline ALT level, $U / L$ & 31 & 22 & 48 & 34 & 24 & 49 & NS \\
\hline ALP level, U/L & 81 & 58 & 101 & 85 & 71 & 106 & NS \\
\hline AST level, U/L & 25 & 18 & 32 & 26 & 20 & 35 & NS \\
\hline GGT level, U/L & 28 & 23 & 34 & 36 & 27 & 47 & .005 \\
\hline $\mathrm{BMI}, \mathrm{kg} / \mathrm{cm}^{2}$ & 44 & 39 & 50 & 40 & 39 & 43 & NS \\
\hline Fat percentage, \% & $54 \%$ & 49 & 56 & $51 \%$ & 46 & 56 & NS \\
\hline Waist circumference, $\mathrm{cm}$ & 122 & 110 & 128 & 123 & 115 & 131 & NS \\
\hline Waist-to-hip ratio & 0.92 & 0.86 & 1.02 & 0.98 & 0.92 & 1.06 & .004 \\
\hline Total cholesterol & 5.2 & 4.4 & 5.7 & 5.2 & 4.6 & 5.7 & NS \\
\hline $\mathrm{HDL}$ & 1.2 & 0.9 & 1.4 & 1.1 & 0.9 & 1.4 & NS \\
\hline LDL & 3.2 & 2.7 & 3.8 & 3.1 & 2.6 & 3.7 & NS \\
\hline Triglycerides & 1.6 & 1.3 & 2.1 & 1.6 & 1.2 & 2.3 & NS \\
\hline Fasting glucose & 5.0 & 4.2 & 6.2 & 4.7 & 4.3 & 5.2 & NS \\
\hline
\end{tabular}

NOTE. A $P$ value of less than .05 was considered as statistically significant.

ALP, alkaline phosphatase; GGT, $\gamma$-glutamyltransferase; HDL, high-density lipoprotein; LDL, low-density lipoprotein. 
Supplementary Table 7. Clinical Characteristics of an Additional 26 Bariatric Patients and 13 Cholecystectomy Patients Included in the Study for Characterization of Adipose Tissue Macrophages

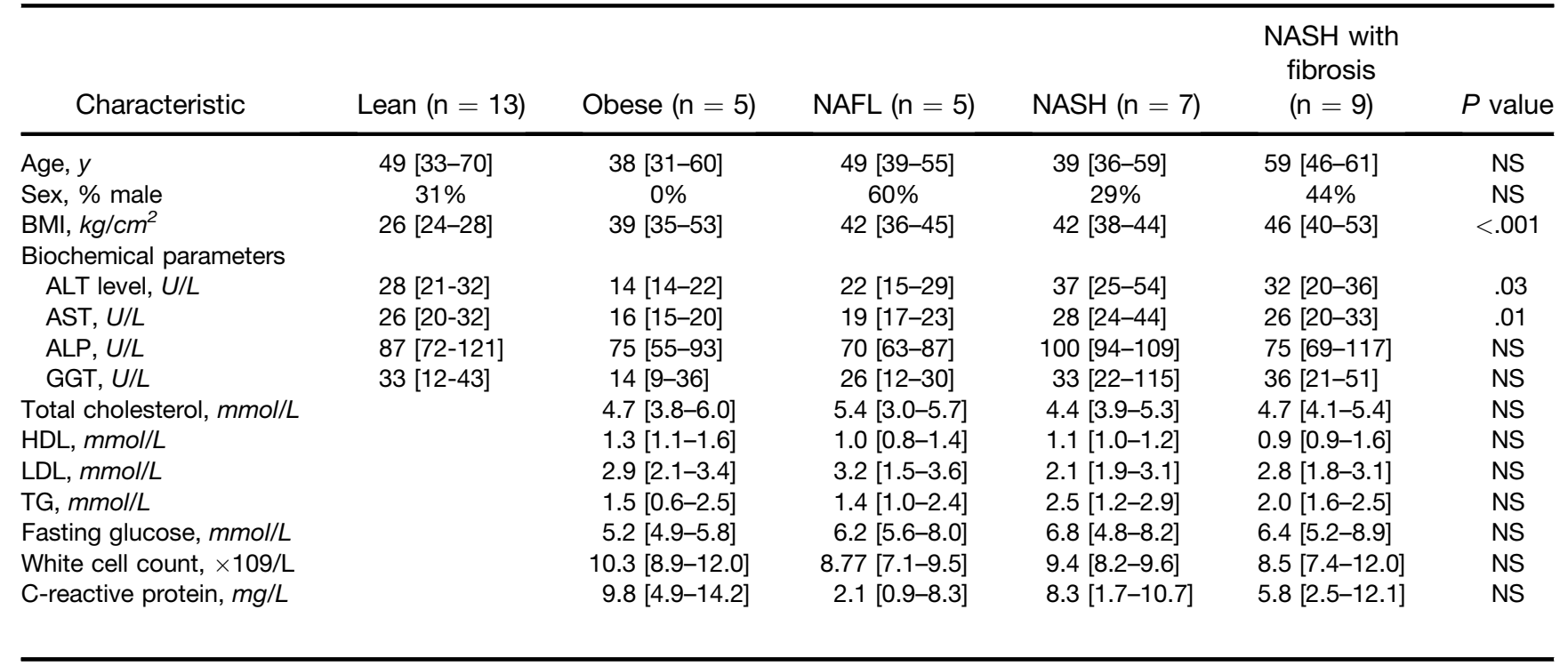

NOTE. Results are expressed as median and interquartile range [25\%-75\%]. The Kruskal-Wallis test was used to compare groups and $P<.05$ was significant.

ALP, alkaline phosphatase; GGT, $\gamma$-glutamyltransferase; HDL, high-density lipoprotein; LDL, low-density lipoprotein; TG, triglyceride.

Supplementary Table 8. Comparison of Clinical Characteristics Between Original and Additional Patient Populations

\begin{tabular}{|c|c|c|c|c|c|c|c|}
\hline \multirow[b]{2}{*}{ Characteristic } & \multicolumn{3}{|c|}{ Original patient cohort $(n=113)$} & \multicolumn{4}{|c|}{ Additional patient cohort $(n=26)$} \\
\hline & Median & $25 \%$ & $75 \%$ & Median & $25 \%$ & $75 \%$ & $P$ value \\
\hline $\begin{array}{l}\text { Number of patients per } \\
\text { group, I-II-III-IV }\end{array}$ & $28-25-41-19$ & & & $5-5-7-9$ & & & \\
\hline Age, $y$ & 42 & 35 & 48 & 49 & 38 & 59 & .03 \\
\hline AST level, $U / L$ & 26 & 20 & 34 & 23 & 17 & 30 & NS \\
\hline GGT level, U/L & 31 & 24 & 44 & 26 & 20 & 48 & NS \\
\hline $\mathrm{BMI}, \mathrm{kg} / \mathrm{cm}^{2}$ & 41 & 39 & 47 & 42 & 38 & 46 & NS \\
\hline Total cholesterol, $\mathrm{mmol} / \mathrm{L}$ & 5.2 & 4.6 & 5.7 & 4.7 & 3.9 & 5.5 & NS \\
\hline $\mathrm{HDL}, \mathrm{mmol} / \mathrm{L}$ & 1.1 & 1.0 & 1.4 & 1.1 & 0.9 & 1.4 & NS \\
\hline LDL, $\mathrm{mmol} / \mathrm{L}$ & 3.2 & 2.7 & 3.8 & 2.7 & 1.8 & 3.2 & .003 \\
\hline
\end{tabular}

ALP, alkaline phosphatase; GGT, $\gamma$-glutamyltransferase; HDL, high-density lipoprotein; LDL, low-density lipoprotein. 
Supplementary Table 9.Detailed Overview of VAT and SAT Microarray Results

\begin{tabular}{|c|c|c|c|c|c|}
\hline \multirow[b]{2}{*}{ Tissue type } & \multicolumn{2}{|c|}{$\begin{array}{l}\text { Differential expression } \\
2 \log \text { value }>1 \text { or }<-1\end{array}$} & \multirow{2}{*}{ Annotated genes } & \multicolumn{2}{|c|}{$\begin{array}{l}\text { Identified pathways } \\
\text { and biological processes }\end{array}$} \\
\hline & Probes & Genes & & KEGG & $\begin{array}{c}\text { GO biological } \\
\text { processes } \\
\text { ( Bonferroni } \\
\text { correction }<.05 \text { ) }\end{array}$ \\
\hline \multicolumn{6}{|l|}{ Visceral adipose tissue } \\
\hline Obese vs NAFL & 16 & 12 & 12 & 0 & 0 \\
\hline NAFL vs NASH & 190 & 139 & 133 & 9 & 19 \\
\hline NASH vs NASH with fibrosis & 280 & 204 & 194 & 3 & 14 \\
\hline \multicolumn{6}{|l|}{ Subcutaneous adipose tissue } \\
\hline Obese vs NAFL & 83 & 58 & 56 & 8 & 8 \\
\hline NAFL vs NASH & 46 & 40 & 37 & 0 & 7 \\
\hline $\mathrm{NASH}$ vs NASH with fibrosis & 104 & 83 & 82 & 9 & 3 \\
\hline
\end{tabular}

GO, gene ontology; KEGG, Kyoto Encyclopedia of Genes and Genomes.

Supplementary Table 10.Analysis of Molecular Pathways and Biological Processes in VAT

\begin{tabular}{|c|c|c|c|}
\hline \multirow{2}{*}{$\begin{array}{l}\text { Visceral adipose tissue } \\
\text { Obese vs NAFL }\end{array}$} & \multicolumn{3}{|c|}{ Number of genes differentially expressed between groups } \\
\hline & \multicolumn{3}{|l|}{12 genes } \\
\hline & \multicolumn{3}{|c|}{ No KEGG pathways or biological processes } \\
\hline \multirow[t]{17}{*}{ NAFL vs NASH } & \multicolumn{3}{|l|}{139 genes } \\
\hline & \multicolumn{2}{|l|}{ KEGG pathways } & $P$ value \\
\hline & hsa04710 & Circadian rhythm & .006 \\
\hline & hsa05219 & Bladder cancer & .007 \\
\hline & hsa05200 & Pathways in cancer & .01 \\
\hline & hsa04621 & NOD-like receptor signaling pathway & .02 \\
\hline & hsa05416 & Viral myocarditis & .03 \\
\hline & hsa04062 & Chemokine signaling pathway & .03 \\
\hline & hsa04060 & Cytokine-cytokine receptor interaction & .03 \\
\hline & hsa04010 & MAPK signaling pathway & .03 \\
\hline & hsa05410 & Hypertrophic cardiomyopathy & .04 \\
\hline & \multicolumn{2}{|c|}{ Top 5 biological processes } & $P$ value \\
\hline & \multicolumn{2}{|c|}{ Behavior } & $8.79 \mathrm{E}-12$ \\
\hline & \multicolumn{2}{|l|}{ Taxis } & $8.45 \mathrm{E}-11$ \\
\hline & \multicolumn{2}{|l|}{ Chemotaxis } & $8.45 \mathrm{E}-11$ \\
\hline & \multicolumn{2}{|c|}{ Response to wounding } & $1.03 \mathrm{E}-10$ \\
\hline & \multicolumn{2}{|c|}{ Inflammatory response } & $2.31 \mathrm{E}-9$ \\
\hline \multirow[t]{11}{*}{ NASH vs NASH with fibrosis } & \multicolumn{3}{|l|}{204 genes } \\
\hline & \multicolumn{2}{|l|}{ KEGG pathways } & $P$ value \\
\hline & hsa04610 & Complement and coagulation cascades & $5.62 \mathrm{E}-6$ \\
\hline & hsa04060 & Cytokine-cytokine receptor interaction & $1.85 \mathrm{E}-5$ \\
\hline & hsa04062 & Chemokine signaling pathway & 005 \\
\hline & \multicolumn{2}{|c|}{ Top 5 biological processes } & $P$ value \\
\hline & \multicolumn{2}{|c|}{ Response to wound healing } & 1.37E-19 \\
\hline & \multicolumn{2}{|c|}{ Inflammatory response } & 4.11E-12 \\
\hline & \multicolumn{2}{|l|}{ Defense response } & $5.28 \mathrm{E}-11$ \\
\hline & \multicolumn{2}{|l|}{ Chemotaxis } & $1.55 \mathrm{E}-9$ \\
\hline & \multicolumn{2}{|l|}{ Taxis } & $1.55 E-9$ \\
\hline
\end{tabular}

NOTE. The number of genes significantly differentially expressed (2logratio $>1$ or $<-1$ ) according to microarray analysis between the histologic groups for VAT were determined and the corresponding molecular pathways and top biological processes were identified by the DAVID Bioinformatics Resource 6.7 program. ${ }^{8}$

KEGG, Kyoto Encyclopedia of Genes and Genomes; MAPK, mitogen-activated protein kinase; NOD, nucleotide-binding oligomerization domain receptors. 
Supplementary Table 11.Analysis of Molecular Pathways and Biological Processes in SAT

\begin{tabular}{|c|c|c|c|}
\hline Subcutaneous AT & \multicolumn{3}{|c|}{ Number of genes differentially expressed between different groups } \\
\hline \multirow[t]{11}{*}{ Obese vs NAFL } & \multicolumn{3}{|l|}{58 genes } \\
\hline & \multicolumn{2}{|l|}{ KEGG pathways } & $P$ value \\
\hline & hsa04514 & Cell adhesion molecules (CAMs) & $2.02 \mathrm{E}-4$ \\
\hline & hsa05332 & Graft-versus-host disease & $5.81 \mathrm{E}-4$ \\
\hline & hsa05416 & Viral myocarditis & .003 \\
\hline & hsa05330 & Allograft rejection & .01 \\
\hline & hsa04940 & Type 1 diabetes mellitus & .01 \\
\hline & hsa04672 & Intestinal immune network & .02 \\
\hline & hsa05320 & Autoimmune thyroid disease & .02 \\
\hline & hsa04612 & Antigen processing and presentation & .05 \\
\hline & \multicolumn{3}{|c|}{ No significant biological processes } \\
\hline \multirow[t]{8}{*}{ NAFL vs NASH } & \multicolumn{3}{|c|}{40 genes } \\
\hline & \multicolumn{3}{|c|}{ No significant KEGG pathways } \\
\hline & \multicolumn{2}{|c|}{ Top 5 biological processes } & $P$ value \\
\hline & \multicolumn{2}{|c|}{ Defense response to fungus } & $1.04 \mathrm{E}-8$ \\
\hline & \multicolumn{2}{|c|}{ Killing of cells of another organism } & $1.45 \mathrm{E}-8$ \\
\hline & \multicolumn{2}{|l|}{ Defense response } & $1.66 \mathrm{E}-8$ \\
\hline & \multicolumn{2}{|l|}{ Cell killing } & $1.52 \mathrm{E}-7$ \\
\hline & \multicolumn{2}{|c|}{ Response to fungus } & $1.52 \mathrm{E}-7$ \\
\hline \multirow[t]{13}{*}{$\mathrm{NASH}$ vs NASH with fibrosis } & \multicolumn{3}{|l|}{83 genes } \\
\hline & \multicolumn{2}{|l|}{ KEGG pathways } & $P$ value \\
\hline & hsa05416 & Viral myocarditis & 9.34E-4 \\
\hline & hsa04514 & Cell adhesion molecules & .001 \\
\hline & hsa04512 & ECM-receptor interaction & .02 \\
\hline & has05330 & Allograft rejection & .02 \\
\hline & hsa05332 & Graft-versus-host disease & .02 \\
\hline & has04940 & Type 1 diabetes mellitus & .03 \\
\hline & hsa04672 & Intestinal immune network & .04 \\
\hline & \multicolumn{2}{|c|}{ Top 5 biological processes } & $P$ value \\
\hline & \multicolumn{2}{|c|}{ Defense response } & $1.46 \mathrm{E}-6$ \\
\hline & \multicolumn{2}{|c|}{ Response to wound healing } & $1.61 \mathrm{E}-6$ \\
\hline & \multicolumn{2}{|c|}{ Killing of cells of another organism } & $4.06 \mathrm{E}-5$ \\
\hline
\end{tabular}

ECM, Extra-cellular matrix; KEGG, Kyoto Encyclopedia of Genes and Genomes.

Supplementary Table 12. Pearson Correlation of Inflammatory Gene Expression Between VAT and SAT

\begin{tabular}{lcc}
\hline & $r$ & $P$ \\
\hline CCL2 & 0.300 & .001 \\
CCL3 & 0.160 & .090 \\
CCL21 & 0.118 & .216 \\
GADD45B & 0.330 & $3.02 \mathrm{E}-04$ \\
IL1RN & 0.362 & $1.08 \mathrm{E}-04$ \\
IL8 & 0.448 & $1.29 \mathrm{E}-06$ \\
JUN & 0.328 & .001 \\
RELN & 0.183 & .050 \\
SERPINE1 & 0.563 & $5.95 \mathrm{E}-11$ \\
& & \\
\hline
\end{tabular}


Supplementary Table 13. Information on the Genes Studied for Classification Model Building as Supplied by NCBI

\begin{tabular}{ll}
\hline \multicolumn{1}{c}{ Gene symbol } & \multicolumn{1}{c}{ Molecular function } \\
\hline$C C L 2$ (chemokine (C-C motif) ligand 2) & Immunoregulatory and inflammatory processes \\
$C C L 3$ (chemokine (C-C motif) ligand 3) & Macrophage inflammatory responses \\
$C C L 21$ (chemokine (C-C motif) ligand 21) & Immunoregulatory and inflammatory processes \\
$D M R T 2^{2}$ (doublesex and mab-3 related transcription factor 2) & Transcription factor \\
$G A D D 45 B$ (growth arrest and DNA-damage-inducible, $\beta$ ) & Regulation of growth and apoptosis \\
IL1RN (interleukin 1-receptor antagonist) & Modulates immune and inflammatory responses \\
$I L 8 / C X C L 8$ (chemokine (C-X-C motif) ligand 8) & Mediators of the inflammatory response \\
JUN/AP-1 (jun proto-oncogene) & Transcription factor \\
RELN (reelin) & Control cell-cell interactions and cell positioning \\
SERPINE1 (serpin peptidase inhibitor, clade E, member 1) & Inhibitor of fibrinolysis \\
ZNF880 (zinc finger protein 880) & Presently unknown \\
&
\end{tabular}

NOTE. Nine genes were selected as classifier for the visceral fat. NCBI accession date: August 5, 2014.

${ }^{a}$ The 2 additional genes DMRT2 and ZNF880 were included as classifiers for subcutaneous fat. 